\title{
Interferometric radar observations of filamented structures due to plasma instabilities and their relation to dynamic auroral rays
}

\author{
T. Grydeland ${ }^{1}$, E. M. Blixt ${ }^{1}$, U. P. Løvhaug ${ }^{1}$, T. Hagfors ${ }^{2}$, C. La Hoz ${ }^{1}$, and T. S. Trondsen ${ }^{3}$ \\ ${ }^{1}$ Dept. of Physics, University of Troms $\varnothing$, N-9037 Troms $\varnothing$, Norway \\ ${ }^{2}$ Max-Planck-Institut für Aeronomie, Max Planck Straße 2, D-37191, Katlenburg-Lindau, Germany \\ ${ }^{3}$ Institute for Space Research, Calgary, Alberta, Canada
}

Received: 14 May 2003 - Revised: 28 August 2003 - Accepted: 4 September 2003 - Published: 2 April 2004

\begin{abstract}
Several explanations have been proposed for Naturally Enhanced ion-acoustic Echoes observed at midand high-latitude Incoherent Scatter observatories. A decisive measure for distinguishing between these explanations is whether or not simultaneously observed up- and downshifted enhancement occur simultaneously, or if they are the result of temporal and/or spatial averaging.

The EISCAT Svalbard Radar has two antennas in the same radar system, which can be used as an interferometer when pointed parallel. In observations from 17 January 2002, between 06:46:10 and 06:46:30 UT, we used this possibility, in combination with direct sampling of the received signals, to yield measurements of "naturally enhanced ion-acoustic echoes" with sufficiently high resolution to resolve such averaging, if any. For the first time, radar interferometry has been employed to estimate the sizes of coherent structures. The observations were coordinated with an image intensified video camera with a narrow field of view. Together, this forms the initial study on the causal relationships between enhanced echoes and fine structure in the auroral activity on sub-kilometer, sub-second scales.

The results confirm that the enhanced echoes originate from very localised regions $(\sim 300 \mathrm{~m}$ perpendicular to the magnetic field at $500 \mathrm{~km}$ altitude) with varying range distribution, and with high time variability $(\approx 200 \mathrm{~ms})$. The corresponding increase in scattering cross section, up to $50 \mathrm{~dB}$ above incoherent scattering, eliminates theoretical explanations based on marginal stability. The simultaneously observed up- and down-shifted enhanced shoulders, when caused by sufficiently narrow structures to be detected by the interferometer technique, originate predominantly from the same volume. These results have significant impact on theories attempting to explain the enhancements, in particular it is found that the ion-electron two-stream mechanism favoured by many authors is an unlikely candidate to explain the observations. The video data has helped establish a clear
\end{abstract}

Correspondence to: T. Grydeland

(tom.grydeland@phys.uit.no) correlation between the enhanced echoes and auroral activity, on sub-second time scales, showing a threshold connection between the auroral intensity and the triggering of the radar enhancements. It appears that the up- and down-shifted enhanced echoes correlate with fine auroral structures in different ways.

Key words. Ionosphere (auroral ionosphere; plasma waves and instabilities) - Radio science (interferometry)

\section{Introduction}

In Incoherent Scatter (IS) radar, most of the scattered power is contained in a narrow (typically double-humped) spectrum centered at the transmitter frequency. This corresponds to scattering from thermally excited highly damped ionacoustic waves travelling towards and away from the radar in the ionospheric plasma.

From time to time, spectra are observed which do not correspond to our understanding of the scattering from a thermal plasma. Strong enhancements of one or both shoulders in the ion-acoustic spectrum occur on very short time scales. These so-called naturally enhanced ion-acoustic echoes were first reported from the Haystack observatory (Foster et al., 1988) and later from the EISCAT UHF and VHF radars (Collis et al., 1991; Rietveld et al., 1991). A survey of more than 5000 hours of observations from 1987 to 1993 was carried out by Rietveld et al. (1996). Enhanced echoes have been observed regularly at the EISCAT Svalbard Radar (ESR), with reports by, for example, Buchert et al. (1999), and Sedgemore-Schulthess et al. (1999). A survey of observations from various observatories and their theoretical explanations has recently been published (Sedgemore-Schulthess and St.-Maurice, 2001).

The echoes from the high-latitude EISCAT radars show significant variations from one five- or ten-second integration period to the next. The observations reported briefly by Grydeland et al. (2003), which uses the same radar data set 
as that discussed herein, demonstrated that the process has a sufficiently high scattering cross section that it can be investigated with much finer temporal resolution than what is possible in normal IS observations, leaving the time scales of this variability available for investigation for the first time. By pointing the two antennas of the EISCAT Svalbard Radar in the same direction and using them as an interferometer, this last study also presented examples of enhancement produced by a localised structure. The study showed an example of enhancement in both ion-acoustic shoulders which was interpreted as having been produced simultaneously and in the same volume.

In this publication, we start with a summary of the relevant theories of enhanced echoes and their relation to optical phenomena in Sect. 2. We explain the experimental methods and techniques used for our observations in Sects. 3, 4 and 5. The high-resolution image intensified video camera and geometry is presented in Sect. 6. The geophysical background for our observations is presented in Sect. 7. A new element in this report is an investigation of the causal relationship of the radar enhancements and aurora. Indeed, we have found for the first time that there is a correlation on sub-second time scales between the auroral intensity and the enhancements of the radar echoes, as described in Sects. 8.5 and 9.2. In Sect. 8 we also show the distribution of sizes obtained from the observations, and demonstrate that the enhancement of both shoulders in a common volume reported by Grydeland et al. (2003) is not a chance event, but rather the typical situation when both up- and down-shifted enhancements are due to scattering from a localised region. A new estimate of the intensity of the radar echoes that takes into account the size of the scattering structures also allows for the conclusion that the plasma processes that produce the echoes must be the result of instability and not of a mere enhancement of the thermal fluctuations near marginal instability, as suggested elsewhere (e.g. Collis et al., 1991). The explanation offered by these authors requires the existence of extremely high current densities, much higher than what has been observed directly, but still within the limits of stability. To produce instability, as required by our observations, the current densities must be higher still. The impact on theories is described in more detail in Sect. 9.3.

\section{Background}

\subsection{Theories}

Sedgemore-Schulthess and St.-Maurice (2001) recently gave an extensive review of prevailing theories and observations of naturally enhanced ion-acoustic echoes. To properly address how these theories are influenced by the observations reported here, however, further discussion is needed. Two broad classes of theories have been offered for these observations: 1) ion-acoustic mode destabilization by counter streaming species, which can be subdivided into ion-electron two-stream instability and ion-ion two-stream instability; and 2) generation of ion-acoustic waves from intense Langmuir waves through nonlinear wave-wave interaction.

The first theory proposed to be the cause of the necessary destabilization of the ion-acoustic mode was the ionelectron two-stream instability. Foster et al. (1988), Collis et al. (1991), Rietveld et al. (1991) and SedgemoreSchulthess et al. (1999) interpret their asymmetric spectra as being due to large thermal electron fluxes contributing to intense field-aligned currents, causing the plasma parameters to approach, but not exceed, the threshold for the onset of the ion-acoustic instability. The field-aligned flow of soft electrons produce parallel electric fields, which again produce the thermal flows. Simulations show that the currents must be of the order of several milliamps per square metre (see, e.g. Rietveld et al., 1991). Although such high values are not observed directly, it is argued that such intense currents may exist in the ionosphere for brief periods of time. This theory cannot account for the simultaneous observation of both up- and down-shifted ion lines at the same time, and Rietveld et al. (1991), therefore, suggested that for such events, one is observing averaging in time over multiple spatially narrow structures. To emphasize that actual instability has not been suggested in this explanation, we will refer to it by the name "two-stream enhancement" in this paper.

Wahlund et al. (1992) argued against the current-driven instability model due to the high current values required, and proposed the ion-ion two-stream instability as the generation mechanism of the enhanced acoustic fluctuations. Based on the study of ion outflow in the topside ionosphere using EISCAT data, asymmetric spectra were often observed during the outflow events. A differential drift between two ion populations, in this case $\mathrm{H}^{+}$and $\mathrm{O}^{+}$ions in the upper ionosphere, with velocities of similar value as their thermal velocity, can lead to an increase in the acoustic fluctuation level to well above the thermal level, or, for sufficiently high relative drifts, ion-acoustic instability. However, such high relative drift is suggested to be unlikely to be present in the lower $F$-region and below, where enhanced echoes are also observed.

Groves et al. (1991) appear to be the first to have suggested a theory involving wave-wave interactions whereby Langmuir waves interact with ion acoustic waves in an unstable situation, drawing free energy from photoelectrons. Forme (1993), more convincingly, suggested that the free energy is supplied by a beam of electrons (i.e. auroral precipitation) which drives unstable Langmuir waves via the bump-in-tail instability (Landau growth), which, in turn, decay into ionacoustic waves and an oppositely directed Langmuir wave. Grydeland et al. (1998), suggested to use the Zakharov equations (Zakharov, 1972) to model the interaction, and Forme (1999) directly solved the Zakharov equations numerically. It is well known (e.g. Hanssen et al., 1992; Robinson, 1997, etc.) that solutions to the Zakharov equations contain all the regimes of wave-wave interactions of a non-magnetised plasma, from parametric instabilities - which produce weak plasma instability (in essence, the mechanism suggested by Groves et al.) - up to strong Langmuir turbulence, well 
known now in the fields of ionospheric RF heating and some kinds of solar radio bursts.

The wave-wave interaction is appealing because it may explain the measurements without requiring large current densities or large differential ion drifts, both of which are problematic. The only condition is that the wave-vector spectrum of the turbulent waves contains the wave vector of the probing radar, which leads to the condition that the electron beam should not be very energetic (tens or at most hundreds of $\mathrm{eV}$, e.g. (Forme, 1993)). If this condition is met, it is not difficult to produce all the spectral features observed in the experiments, such as one or the other or both of the shoulders enhanced and varying in altitude, because the 3-dimensionality of the wave-vector spectrum leaves considerable freedom to attain the matching condition for the radar scattering. The inhomogeneity of the medium, combined with the turbulent variability, may alternately favour one wave or the other or both, varying in time and space. What is even more interesting, in the light of our measurements, is that these theories can explain the simultaneous enhancements of both shoulders in the same volume. They are just enhanced ion-acoustic waves travelling in opposite directions and generated in different cascades of the instability. The underlying wave-wave interaction is a backscatter mode, each newly produced ion acoustic wave (higher order cascade) having $\boldsymbol{k}$-vector directed nearly oppositely to the wave in the previous cascade.

\subsection{Relation to optical phenomena}

If the enhanced echoes are generated by a two-stream instability, or by cascading Langmuir waves, the common factor is the existence of electrons streaming along the magnetic field. It is well known that auroral activity is also associated with such streaming electrons. These are found either in the upward leg of the field-aligned current, where electrons are precipitating, or in the adjacent downward leg of the current circuit, where upward going electrons are carrying the current. Despite this apparent connection between auroral activity and naturally enhanced ion-acoustic echoes, few studies have been made of the detailed relation, possibly due to the limited resolution of IS radar measurements.

Collis et al. (1991) noticed that red aurora was always present during their observations of enhanced echoes, and Sedgemore-Schulthess et al. (1999) also observed enhanced echoes at the equatorward edge of red aurora. The latter also used a narrow-angle video camera, and closer inspections reveals the existence of small-scale auroral forms, but no conclusions were drawn concerning the detailed connections between the auroral activity and the enhanced radar echoes. Forme et al. (1995) associated their enhanced radar echoes with auroral precipitation at less than $\mathrm{keV}$ energies by inverting the electron density profiles measured by the radar. Cabrit et al. (1996) used the two antennas at EISCAT in Troms $\varnothing$ to deduce the plasma parameters simultaneously with the observation of an enhanced echo, and found that a discrete auroral arc passed through the radar beam. Further analysis allowed them to conclude that enhanced echoes are preferably located on the edge of auroral arcs. All four aforementioned studies were strongly limited by the $10 \mathrm{~s}$ integration time inherent in the radar observations.

Image intensified video cameras have established a wealth of small-scale auroral forms (Maggs and Davis, 1968; Trondsen and Cogger, 2001), and by using similar equipment as that used by Trondsen and Cogger (2001), we have for the first time been able to make close comparisons between the activity of small-scale auroral structures and the activity of enhanced echoes. This was made possible due to the very high temporal resolution of the radar experiment.

\section{The Radar and the experiment}

The EISCAT Svalbard Radar is situated at $78^{\circ} 9^{\prime} \mathrm{N}, 16^{\circ} 2^{\prime} \mathrm{E}$, near the community of Longyearbyen on the Spitzbergen Island of the Svalbard archipelago. The location puts the radar in a region often covered by the arctic cusp, and with a view into the polar cap to the north, as well as an opportunity for overlap with the mainland EISCAT radars when looking to the south. A description of the instrument and its capabilities is given by Wannberg et al. (1997), with some updated information by Grydeland et al. (2002).

Unlike other IS radars, the ESR has two antennas in the same radar system, one fully steerable with a diameter of $32 \mathrm{~m}$ and one fixed with a diameter of $42 \mathrm{~m}$, and a line of sight along the local magnetic field at an azimuth of $181.0^{\circ}$ and an elevation of $81.6^{\circ}$. The distance between the centres of the two antennas is $128 \mathrm{~m}$, which corresponds to 213.481 wavelengths of a radio wave at the $500 \mathrm{MHz}$ frequency of the radar. The baseline between the two antennas is at an azimuth of $68.3^{\circ}$. The existence of the two antennas offers the opportunity of using an interferometer reception technique, one which has proved to be very useful in the case presented below.

In the receiver system of the ESR, the $500 \mathrm{MHz}$ radar signal is first mixed with $430 \mathrm{MHz}$ in the antenna, and bandpass filtered to $70 \pm 2.5 \mathrm{MHz}$. This signal is passed to the control room. The signal is then mixed with $77.5 \mathrm{MHz}$ and filtered to $7.5 \pm 1.9 \mathrm{MHz}$. Mixing the signal "from above" like this inverts the frequency axis. Normally, this signal is then sampled at $10 \mathrm{MHz}$ and proceeds through the ESR's own digital receiver chain. In addition, the signal from both antennas, after the mix to $7.5 \mathrm{MHz}$, was split off and sampled separately using a portable MIDAS-W type receiving system (Holt et al., 2000).

For these observations, the long pulse experiment gup 0 , described briefly here, was used. In this experiment, three pulses of $360 \mu \mathrm{s}$ and one of $180 \mu \mathrm{s}$ are transmitted directly after each other, all on different frequencies, before receiving on all four channels. In the next transmit/receive period (called interpulse period or IPP), four different frequencies are used for a total of eight frequencies. The fixed $42 \mathrm{~m}$ antenna was used to transmit and receive in the ESR system. The bandwidth and sampling rate of the MIDAS-W receiver 


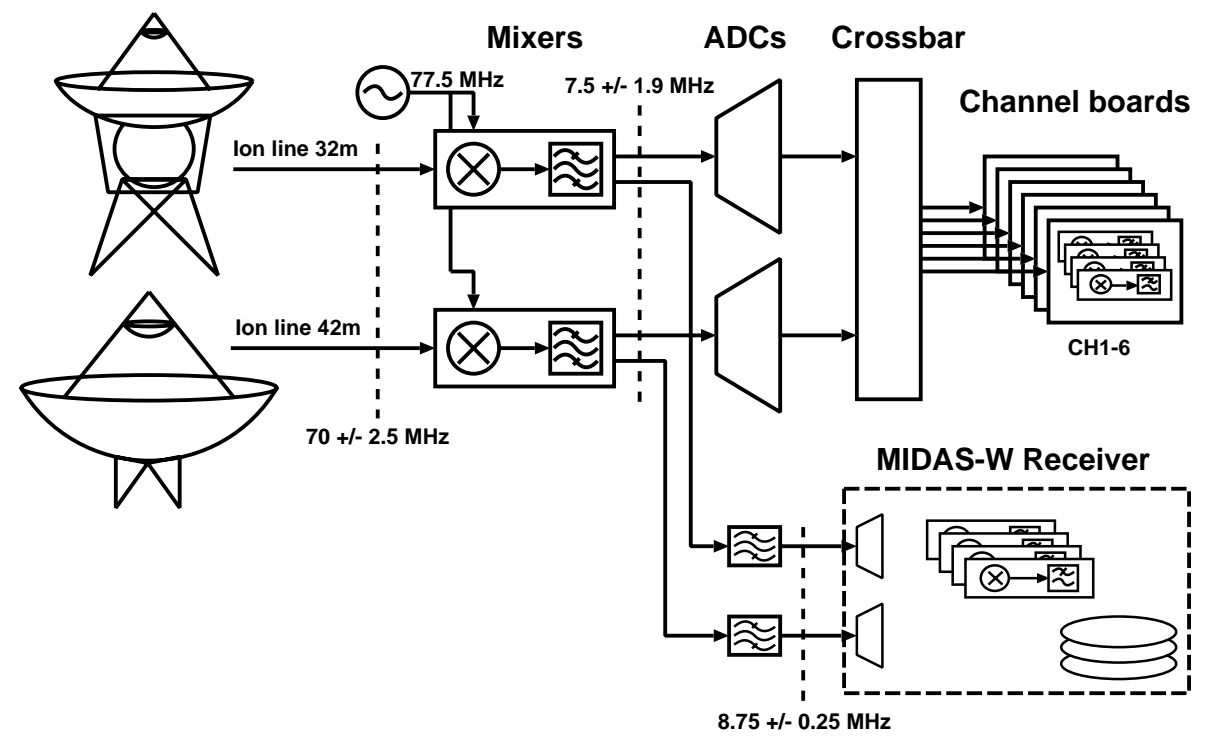

Fig. 1. Block diagram of the ESR ana$\log$ /digital receiver showing the path of the signal through the system and how the MIDAS-W receiver is connected to the existing system. The vertical dashed lines at various points show the center frequency and bandwidth of the signal at those stages was sufficient to record all eight frequencies used in this experiment.

The signal path through the ESR's analog/digital receiver and how the MIDAS-W receiver is connected to the system is shown in Fig. 1. Although the six channel boards can be used for the signal from the two antennas in any combination, they are all used for the signal from the $42 \mathrm{~m}$ antenna in our case.

\section{The interferometric technique}

Incoherent scatter radars have been used for interferometry previously, For instance, Farley et al. (1981) used interferometry between different quadrants of the Jicamarca array to infer velocities of travelling eddies in the equatorial electrojet, and an experimental mode building on this technique is now in regular use at this observatory (e.g. Kudeki et al., 1999; Chau and Woodman, 2001). In these and related publications, a simple expression for the coherence is used. When the two receiving antennas have different gains or the width of the scatterer is not negligible compared to the antenna beam width, a more careful treatment of the effect of antenna gain patterns might be necessary, leading to a more complicated expression. This more careful treatment, which can yield more exact size estimates of the coherent structures, follows below.

Place a coordinate system with the origin in the transmitting antenna, the $\mathrm{z}$-axis in its pointing direction and $\mathrm{x}$-axis along the direction toward the receive-only antenna in the plane perpendicular to $z$. (A component parallel to $z$ results in a constant phase difference, which does not influence our result.) All linear dimensions are expressed in units of the wavelength, which is valid for small relative bandwidths. In the far field, the gain patterns of the two antennas are denoted by $G_{\mathrm{tx}}$ and $G_{\mathrm{rx}}$, respectively. The spatial part of the electric field incident on the scattering volume is given by

$E_{\text {inc }}=\frac{E_{0} R_{0}}{R} G_{\mathrm{tx}}(x / z, y / z) \exp \{-2 \pi i R\}$,

where $R=\sqrt{x^{2}+y^{2}+z^{2}}$ is the distance from the transmitting antenna, and $E_{0}$ is the field at a distance $R_{0}$. The source term for the scattering is obtained by multiplying this expression with a term proportional to the electron density fluctuations, $n(x, y, z)$, and the scattered field is obtained by integrating the source term modified by the reverse distance over the scattering volume.

Introduce the function $g(z)$ centered at zero which expresses the localizing property of a well-designed radar experiment, and use it to limit scattering to a volume centered at $Z_{0}$, where $Z_{0} \gg 1, x, y$. With these inequalities, $z$ and $R$ can be replaced with $Z_{0}$, except where phase is involved. Then define

$n_{k}\left(x, y ; Z_{0}\right)=\int g\left(z-Z_{0}\right) n(x, y, z) e^{-4 \pi i z} d z$.

With this definition, the triple integral over scattering volume reduces to a double integral over transverse directions. The phase factor in the integral is valid for backscattering (i.e. the receivers must be close to the transmitter), and selects only structures of a certain $\boldsymbol{k}$. The scattered field in the aperture plane can now be expressed as

$$
\begin{aligned}
E_{\mathrm{sc}}(x, y)= & \frac{C}{Z_{0}^{2}} \iint G_{\mathrm{tx}}\left(x^{\prime} / Z_{0}, y^{\prime} / Z_{0}\right) n_{\boldsymbol{k}}\left(x^{\prime}, y^{\prime} ; Z_{0}\right) \\
& \exp \left[-2 \pi i \frac{x^{\prime 2}+y^{\prime 2}}{Z_{0}}+2 \pi i \frac{x x^{\prime}+y y^{\prime}}{Z_{0}}\right] \\
& \exp \left[-2 \pi i \frac{x^{2}+y^{2}}{2 Z_{0}}\right] d x^{\prime} d y^{\prime},
\end{aligned}
$$


where the constant $C$ describes the scattering strength of the medium and includes among others the scattering cross section of an electron and the factor $E_{0} R_{0}$.

The received signals in an arbitrary antenna on the ground can now be found by integrating this field over the antenna aperture, which, in the far field approximation, is equivalent to multiplying the expression under the integrals with the receiving antenna pattern and taking the phase from its centre.

The received signal in the transmitting antenna is given by

$f_{\mathrm{tx}}=\frac{C}{Z_{0}^{2}} \iint n_{\boldsymbol{k}}\left(x, y ; Z_{0}\right) G_{\mathrm{tx}}^{2} e^{-2 \pi i\left(x^{2}+y^{2}\right) / Z_{0}} d x d y$.

And for the receive-only antenna, with gain pattern $G_{\mathrm{rx}}$ and displaced distance $(x, y)=(A, 0)$,

$f_{\mathrm{rx}}=\frac{C}{Z_{0}^{2}} \iint n_{k}\left(x, y ; Z_{0}\right) G_{\mathrm{tx}} G_{\mathrm{rx}} e^{-2 \pi i\left(x^{2}+y^{2}-A x\right) / Z_{0}} d x d y$,

where the constant phase factor $e^{-\pi i A^{2} / Z_{0}}$ has been discarded.

From expressions (4) and (5), form the cross-product and ensemble average, which gives an expression for the complex spatial cross-correlation of the scattering received in the two antennas. Assume homogeneity for the spatial correlations,

$\left\langle n_{\boldsymbol{k}}\left(x, y ; Z_{0}\right) n_{\boldsymbol{k}}^{*}\left(x^{\prime}, y^{\prime} ; Z_{0}\right)\right\rangle\left\langle|\Delta n|^{2}\right\rangle \delta\left(x-x^{\prime}\right) \delta\left(y-y^{\prime}\right)$,

introduce the angles of integration $\theta_{x}=x / Z_{0}, \theta_{y}=y / Z_{0}$, and obtain

$$
\begin{aligned}
\left\langle f_{\mathrm{tx}} f_{\mathrm{rx}}^{*}\right\rangle= & \frac{C^{2}}{Z_{0}^{2}} \iint\left\langle|\Delta n|^{2}\left(\theta_{x}, \theta_{y}\right)\right\rangle e^{-2 \pi i A \theta_{x}} \\
& \times G_{\mathrm{tx}}\left(\theta_{x}, \theta_{y}\right)^{3} G_{\mathrm{rx}}\left(\theta_{x}, \theta_{y}\right) d \theta_{x} d \theta_{y} .
\end{aligned}
$$

Assuming for mathematical convenience Gaussian gain patterns for the two antennas,

$G_{\mathrm{tx}, \mathrm{rx}}\left(\theta_{x}, \theta_{y}\right)=\exp \left[-\frac{\theta_{x}^{2}+\theta_{y}^{2}}{2 \sigma_{\mathrm{tx}, \mathrm{rx}}}\right]$,

and likewise that a discrete scatterer centered at $\left(\theta_{x o}, \theta_{y o}\right)$ has a Gaussian shape with unequal widths in the $\mathrm{x}$ - and $\mathrm{y}$ directions, $\sigma_{x}$ and $\sigma_{y}$, respectively,

$\left\langle\left|\Delta n\left(\theta_{x}, \theta_{y}\right)\right|^{2}\right\rangle=\exp \left[-\frac{\left(\theta_{x}-\theta_{x o}\right)^{2}}{2 \sigma_{x}^{2}}-\frac{\left(\theta_{y}-\theta_{y o}\right)^{2}}{2 \sigma_{y}^{2}}\right]$,

the integrals in (7) can be evaluated to obtain

$$
\begin{aligned}
\left\langle f_{\mathrm{tx}} f_{\mathrm{rx}}^{*}\right\rangle= & \frac{C^{2}}{Z_{0}^{2}} \exp \left[-\frac{1}{2} \frac{D_{x}^{2}}{\Sigma^{2}} \frac{\theta_{x o}^{2}}{\sigma_{x}^{2}}-\frac{1}{2} \frac{D_{y}^{2}}{\Sigma^{2}} \frac{\theta_{y o}^{2}}{\sigma_{y}^{2}}\right] \\
& \times \exp \left[-\frac{1}{2}(2 \pi A)^{2} D_{x}^{2}\right] e^{-2 \pi i A \theta_{x o} D_{x}^{2} / \sigma_{x}^{2}}
\end{aligned}
$$

where

$$
\frac{1}{\Sigma^{2}}=\frac{3}{\sigma_{\mathrm{tx}}^{2}}+\frac{1}{\sigma_{\mathrm{rx}}^{2}}, \quad \frac{1}{D_{x, y}^{2}}=\frac{1}{\Sigma^{2}}+\frac{1}{\sigma_{x, y}^{2}} .
$$

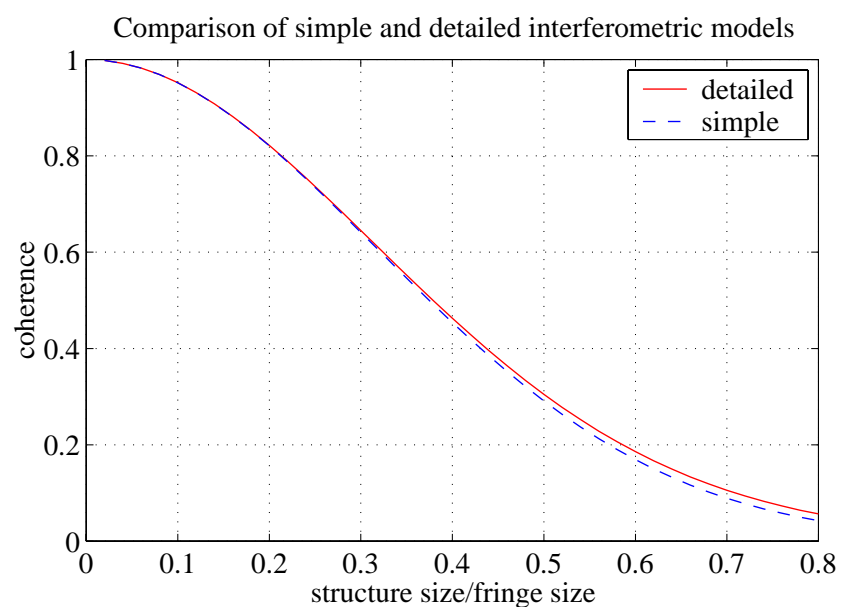

Fig. 2. Comparison of coherence computed using point antennas (dashed line) and Gaussian antennas, Eq. (12) (solid line), where the beam widths and separation of the EISCAT Svalbard Radar antennas have been used. The fringe size $(=1 / 2 A)$ corresponds to $234 \mathrm{~m}$ at $100 \mathrm{~km}$ range.

(Note the change in sign of the $(2 \pi A)^{2}$ term from Eq. (2) in (Grydeland et al., 2003).) For the auto-correlation of the signal in each antenna, repeating steps (7)-(10)leads to

$$
\begin{aligned}
& \left\langle\left|f_{\mathrm{rx}}\right|^{2}\right\rangle=\frac{C^{2}}{Z_{0}^{2}} \exp \left[-\frac{1}{2} \frac{D_{\mathrm{rx}, x}^{2}}{\Sigma_{\mathrm{rx}}^{2}} \frac{\theta_{x 0}^{2}}{\sigma_{x}^{2}}-\frac{1}{2} \frac{D_{\mathrm{rx}, y}^{2}}{\Sigma_{\mathrm{rx}}^{2}} \frac{\theta_{y 0}^{2}}{\sigma_{y}^{2}}\right], \\
& \frac{1}{\Sigma_{\mathrm{rx}}^{2}}=\frac{2}{\sigma_{\mathrm{tx}}^{2}}+\frac{2}{\sigma_{\mathrm{rx}}^{2}}, \quad \frac{1}{D_{\mathrm{rx}, x, y}^{2}}=\frac{1}{\Sigma_{\mathrm{rx}}^{2}}+\frac{1}{\sigma_{x, y}^{2}},
\end{aligned}
$$

with corresponding result for $\left\langle\left|f_{\mathrm{tx}}\right|^{2}\right\rangle$. Normalizing the crosscorrelation by the geometrical mean of the auto-correlations produces the expression for coherence which can be compared to the coherence spectrum computed as explained in the next section;

$\rho=\frac{\left\langle f_{\mathrm{tx}} f_{\mathrm{rx}}^{*}\right\rangle}{\sqrt{\left\langle\left|f_{\mathrm{tx}}\right|^{2}\right\rangle\left\langle\left|f_{\mathrm{rx}}\right|^{2}\right\rangle}}$.

In this expression, the coherence for a structure of a given size does not vary much when moved across the beam, as expected. For the ESR, $A=213.481, \sigma_{\mathrm{tx}}=0.01125$, $\sigma_{\mathrm{rx}}=0.01500$, and for these parameters, coherence in the detectable range computed by the more general expression deviates less than $5 \%$ from that computed using Eq. (8) by Farley et al. (1981), valid in the limit where the receiving antennas have equal gain patterns and the scatterer is much narrower than the antenna beam. Coherence computed using the two expressions is compared in Fig. 2, using parameters for the ESR.

Only scatterers which scatter with the same phase difference from every pulse will stand out from the noise after integration, and only scatterers significantly smaller than the beam width will result in coherence at a detectable level. Although only the size along the baseline $\mathrm{x}$-direction matters in expression (10), a structure elongated along $y$ and with 


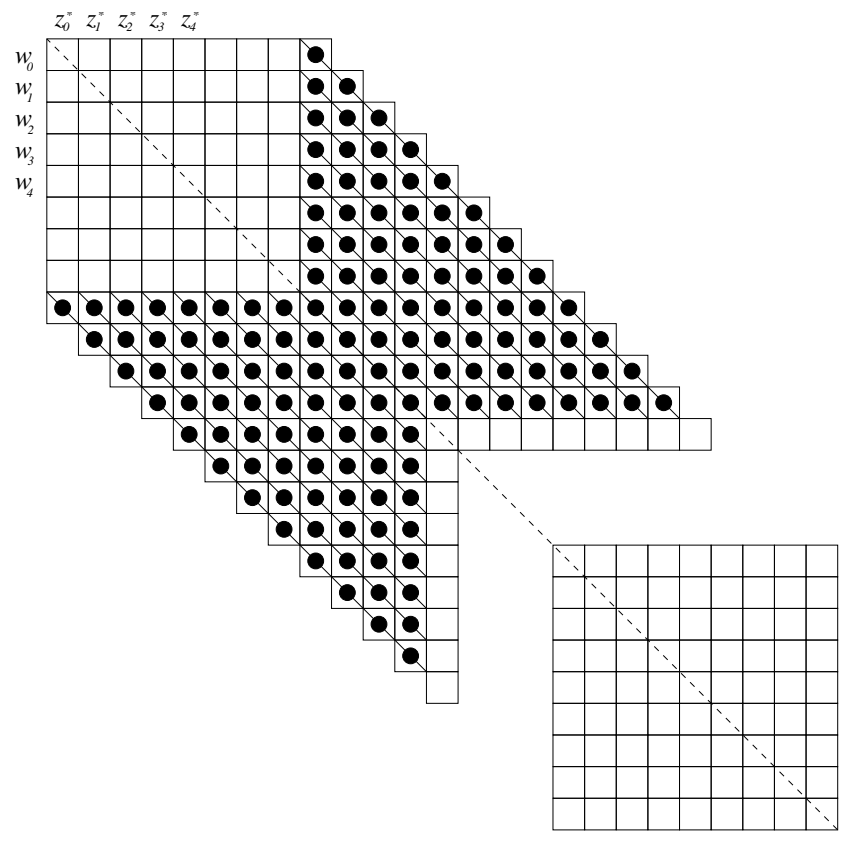

Fig. 3. A small cross-lag profile matrix (XLPM) with eight nonzero lags in either direction. The points used for estimating the cross-correlation function (XCF) for a single range are marked; four lag zero points are used here. The points along each diagonal are averaged to form the estimate of the cross-correlation at that lag value.

even a small angle with the perpendicular to the baseline, would have a larger size along $x$ and hence, a lower coherence. Therefore, we expect observable coherence to arise only from structures which are localised in both horizontal directions, that is, near cylindrical.

\section{Radar data processing}

As can be seen from the above, we were interested in the powerspectra from both antennas as well as their crossspectrum. As is common for IS radar data, spectral information is obtained by way of correlation functions, as this achieves better range resolution without unreasonable limitations on spectral resolution. For auto-correlation function (ACF) estimates, a lag profile matrix (LPM) is produced (Turunen and Silén, 1984, called cross-product or UNIPROG matrix here), while for cross-correlation function (XCF) estimates, we have introduced the cross-lag profile matrix (XLPM) where the elements are

$X_{i, j}=w_{i} z_{j}^{*}$,

where $w$ and $z$ are the signals from the two antennas, and both positive and negative lags must now be computed.

The trapezoidal rule used to extract ACF estimates from an LPM (Turunen and Silén, 1984; Nygren, 1996, Sect. 5.8) has similarly been extended to negative lags for XCF estimates, as illustrated in Fig. 3.

\subsection{The effect of windowing in spectral estimation}

Although fitting for plasma parameters is usually done on correlation data, most human observers find spectra more informative than correlation functions. Powerspectrum or cross-spectrum estimates can be obtained by Fourier transforming the correlation function estimates, but the resulting spectrum is subject to distortions, depending on the lag windowing function employed (Percival and Walden, 1993, Sects. 6.7 and 6.8). If the direct spectral estimator is an unbiased estimator of the true powerspectrum $S(f)$ and the windowed estimator is denoted by $\hat{S}^{(w)}(f)$, then the effect of the window is a smoothing in the spectral domain

$E\left\{\hat{S}^{(w)}(f)\right\} \approx \int_{-f_{N}}^{f_{N}} H\left(f-f^{\prime}\right) S\left(f^{\prime}\right) d f^{\prime}$,

where $E\{\cdot\}$ signifies the expectation value and $H$ is the Fourier transform of the lag window used, called a kernel in this context. Fourier transforming the correlation function estimates directly corresponds to using a rectangular window which has a $\sin (x) / x$ kernel. This kernel has slowly decaying sidelobes, which give rise to an effect called "spectral leakage", where the estimation of low-power frequencies is influenced by high-power frequencies. In Fig. 4, this effect is identified as oscillations in the powerspectrum. In the coherence (lower right panel), windowing effects from the crossspectrum and, through the normalization, the powerspectra, can be seen. The Hanning window's kernel has fast decaying sidelobes, at the cost of a wider mainlobe. This gives estimates with good protection against spectral leakage at the cost of a slight loss of spectral resolution.

While the effect of improper windowing is not very obvious in the powerspectra, except in the low-power frequencies, the effect on the coherence estimation is profound. In the lower right panel of Fig. 4, the coherence curve for rectangular windows (the broken line) shows large oscillations and it exceeds unity on occasions, both of which are unphysical. Using a well-behaved window eliminates the unphysical behaviour.

\subsection{Practical implementation}

The signal from each antenna is passed through a $500 \mathrm{kHz}$ wide band-pass filter centered at $8.75 \mathrm{MHz}$, it is sampled at $1 \mathrm{MHz}$; and recorded separately from each of the two antennas. Sampling this aliasing zone inverts the frequency band again, offsetting the reversal from the mix with $77.5 \mathrm{MHz}$. The resulting time series contains all eight frequencies used in the experiment, four in each IPP. During off-line processing, each of the eight frequencies is mixed to baseband, low-pass filtered and decimated to $20 \mu$ s complex samples, which corresponds to $50 \mathrm{kHz}$ bandwidth. From the reducedrate data vectors, LPMs for the signal from each antenna and their XLPM are formed. Data from the different pulses has been integrated together.

At the end of an integration period, ACFs and XCFs are formed, as indicated above, and stored to file. Nine points 

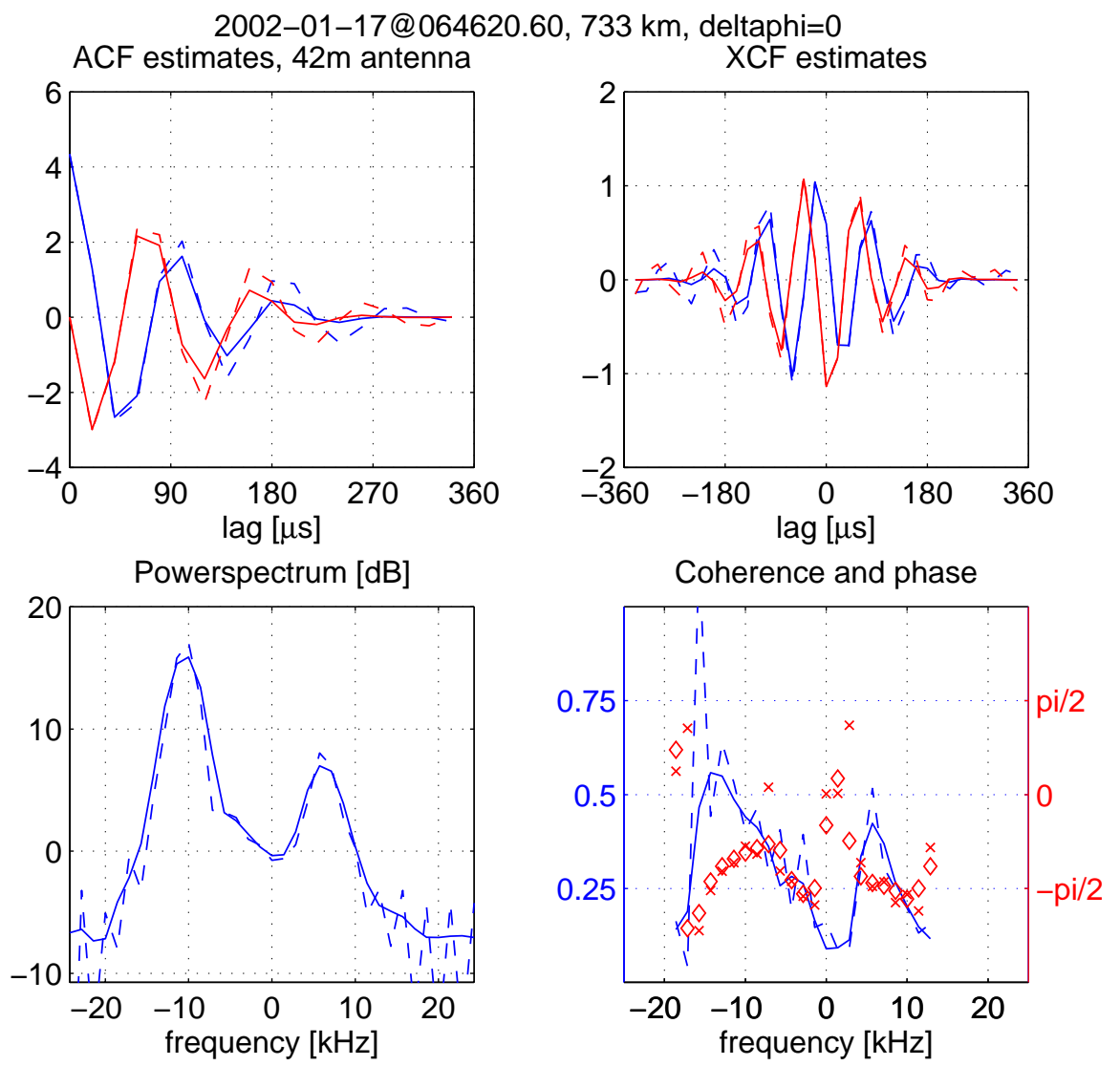

Fig. 4. The effects obtained using windowing on the correlation functions, these examples are from the $0.2 \mathrm{~s}$ integration starting on 06:46:20.60 UT, for the range gate centered at $733 \mathrm{~km}$. The top two panels show correlation function estimates computed from the measurements, real parts in blue, imaginary parts in red. Broken lines are the original estimates, solid lines are after windowing has been applied. The top left panel shows the ACF estimate obtained with the $42 \mathrm{~m}$ antenna, while the top right panel shows the XCF estimate. The bottom left panel shows the resulting powerspectra, using rectangular (broken line) and Hanning (solid line) windows, while the bottom right panel shows the resulting coherence (blue lines) and phase (red markers) for rectangular window (broken lines, crosses) and Hanning window (solid lines, diamonds).

along the main diagonal are used for each range gate, so that each gate has a range coverage about half the length of the $360 \mu$ s pulse.

Powerspectra are computed from ACF estimates by making them periodic, then applying the Hanning window and Fourier Transforming. An example of a power spectrum is shown in the lower left panel of Fig. 4. Cross-spectra are formed the same way, except that they are not made periodic. Coherence is then computed by normalizing the crossspectrum by the geometrical mean of the powerspectra

$\hat{\rho}=\frac{\hat{S}_{\mathrm{tx}, \mathrm{rx}}}{\sqrt{\hat{S}_{\mathrm{tx}, \mathrm{tx}} \hat{S}_{\mathrm{rx}, \mathrm{rx}}}}$.

The normalization is done frequency by frequency. An example of a coherence spectrum is shown in the lower right panel of Fig. 4. High coherence indicates scattering from a spatially limited structure, as discussed in Sect. 4. The magnitude of the observed coherence is a measure of the size of the scattering structure in the direction of the baseline, while the phase corresponds to its position along the baseline, although this correspondence is not unique.

Sky noise is present in the signal from both antennas, and no attempt is made at correcting for this in the present work. Since noise in each signal correlates with itself, but they are mutually uncorrelated, the presence of noise, through the normalization in Eq. (15), leads to a lowering of the observed coherence relative to the maximum observable coherence for a structure of a given size. This effect is more severe for low signal-to-noise ratios. As a result, structures on the edge of the antenna pattern will appear with lower coherences than corresponding structures centered in the beam. Multiple localised scatterers at the same range and frequency will also reduce the observed coherence. As discussed by Grydeland et al. (2003), this means that estimating the horizontal size of a scattering structure through Eq. (12) without considering these sources of error results in overestimating the structure size. Our size estimates are, therefore, upper thresholds of actual sizes. 


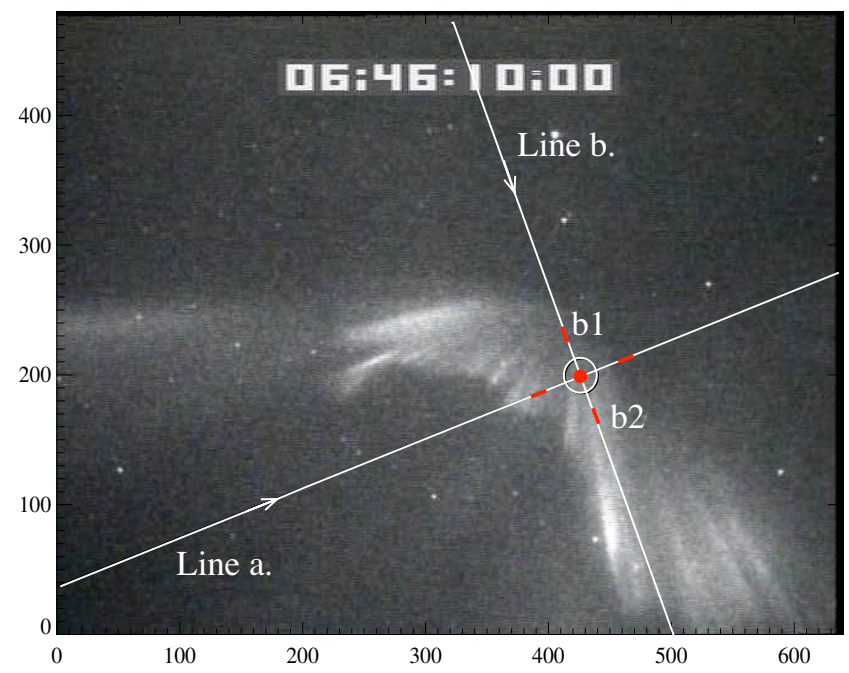

Fig. 5. An image from the camera from 06:46:10 UT, north is upward and east is to the left. The white circle near the center of the image corresponds to the radar beam mapped to $105 \mathrm{~km}$ altitude. The white line (line a) corresponds to the baseline joining the two radar antennas, with the arrow indicating the positive direction. The other line is drawn $90^{\circ}$ towards the base line. The red areas corresponds to regions where we have picked time series of normalised light intensity (per pixel) which has been correlated with the radar intensity time series, as shown in Fig. 14.

\section{The camera}

To supplement the radar observations of enhanced echoes, an image intensified video camera was installed at the Auroral Station (Sigernes et al., 2002), $7 \mathrm{~km}$ northwest of the radar site. This allowed for the investigation of the detailed relationship between auroral activity and the dynamics of the enhanced echoes.

\subsection{Instrument description}

The University of Calgary Portable Auroral Imager (PAI) was designed for high spatial and temporal resolution optical measurements of auroral phenomena (Trondsen, 1989). High sensitivity and linear response is achieved through the use of an $18 \mathrm{~mm}$ Generation III image intensifier, with an extended-blue response photocathode fiberoptically coupled to a Charge Coupled Device (CCD). The imager is equipped with a standard C-mount lens adapter, allowing for the use of a wide range of optical assemblies, from telescopes to allsky lenses. Output data adheres to the RS-170(NTSC) video signal standard, and is stored for later digitization using high resolution (S-VHS) analog video recording equipment. Each video frame is imprinted with an invisible time stamp, using information derived from Global Positioning System (GPS) satellites. The time stamp can be displayed in the image frame during later playback of data, as seen in Fig. 5.

The imager was run during the January 2002 campaign from the Auroral Station. Ideally, the imager should have been run from the ESR site, however, strong interference from the radar itself made that impossible. Subsequent campaigns will have the imager mounted at the ESR site.

Using stars as reference points, the camera head was pointed toward the local magnetic zenith. The exact pointing was $145^{\circ} 23^{\prime}$ azimuth, $82^{\circ} 16.25^{\prime}$ elevation. A $25 \mathrm{~mm}$ focal length, $f / 1.4$ lens provided a viewing angle of about $31^{\circ}$ by $23^{\circ}$, yielding a field of view of $55 \mathrm{~km}$ by $42 \mathrm{~km}$ at $105 \mathrm{~km}$ altitude, which is the most frequent altitude for aurora to occur. The imager's spatial resolution is on the order of 150 meters at $105 \mathrm{~km}$ altitude, limited by the image intensifier's point spread function. The imager outputs 30 frames per second, each frame consisting of two interlaced fields. Each field is formed in $1 / 60 \mathrm{~s}$, yielding a temporal resolution of $1 / 60 \mathrm{~s}=0.017 \mathrm{~s}$. Image intensifier gain was manually controlled and recorded. No optical filtering was performed during the experiment, so as to maximise the amount of light available to form an image. The spectral response of the imager was thus determined by the photocathode, which has better than $10 \%$ quantum efficiency in the range 420 $880 \mathrm{~nm}$, with $30 \%$ quantum efficiency at $557.7 \mathrm{~nm}$. Because we used no filtering, and the response of the camera varies with wavelength, the absolute brightnesses of auroral emissions could not be determined.

In all images presented here, the magnetic zenith is close to the center of the frame, with the top and right being to the north and west, respectively. In Fig. 5 we map the half power radar beam from the $42 \mathrm{~m}$ antenna up to $105 \mathrm{~km}$ altitude, Aurora is of course extended in altitude, and soft-particle precipitation generates aurora at an higher altitude than $105 \mathrm{~km}$. Other mapping altitudes have been used, with no significant change in the overall results.

\subsection{Caveats}

Because the radar and imager were not closely colocated, there arises an ambiguity in the mapping of optical features to the location of the radar beam. Figure 6 shows a simple sketch of the radar/imager setup. The drawing is not to scale, and the separation of the two instruments is exaggerated. North is to the left, and notice that only one of the two radar antennas is shown. The field of view of the radar (hatched red lines) is much smaller than that of the camera (hatched blue lines). The thick blue horizontal line corresponds to the imager's field of view at an altitude $h$, and the red thick line is the radar beam mapped to that same altitude. An auroral arc situated in the position of "Arc a." is located within the radar beam, and only the lower part of the arc will appear as inside the radar beam when mapped to $h$, as viewed from the camera. However, an arc situated south of the radar beam, such that position $b$. will appear to be within the radar beam when viewed from the imager.

This ambiguity can affect our results, but it is difficult to estimate how much and in what way. To avoid such ambiguity the imager must be colocated with the radar, but as previously mentioned, interference problems hindered this during this campaign. It should be noted that the situation drawn in Fig. 6 is exaggerated, and that the setup still allows for 


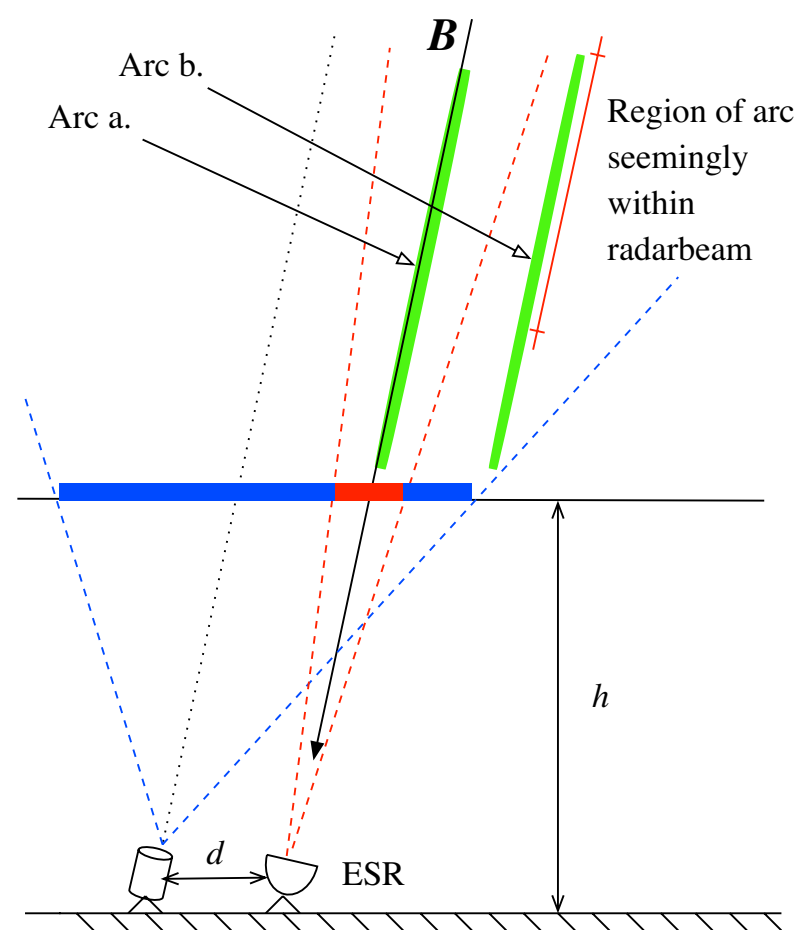

Fig. 6. Simple drawing depicting the experimental setup, highlighting the difficulties due to the distance between the two instruments. The camera (drawn as a cylinder) is located $7 \mathrm{~km}$ from the radar, almost directly north of it. Both the camera and the radar are pointing along the field.

far more detailed correlation studies than previously possible. Furthermore, others (e.g. Sedgemore-Schulthess et al., 1999) have used the exact same configuration without discussing the consequences of the unfavorable geometry.

\section{Geophysical conditions}

The geomagnetic conditions were moderately active, with a $K_{p}$-index of 3 in the hours before the event. The magnetometer data for Longyearbyen (not shown) show that a small substorm commenced at 03:00 UT, but it is on its way to settle when our enhanced echoes are observed. However, a spike in the D-component is pronounced during our time of interest. Data from the University of Alaska MSP (Meridian Scanning Photometer) located at the Auforal Station and operated by the University Centre on Svalbard (UNIS) is shown in Fig. 7. From the top to the bottom panels in the figure, the $630.0 \mathrm{~nm}, 427.8 \mathrm{~nm}, 557.7 \mathrm{~nm}, 488.1 \mathrm{~nm}$ and $844.6 \mathrm{~nm}$ intensities, respectively, are displayed from 02:00 to 10:00 UT. At the time of the observation of our enhanced echoes, (around 06:46 UT), signatures of soft particle precipitation $(630.0 \mathrm{~nm})$ are seen in the ESR pointing direction, which is at an elevation of $98^{\circ}$ for the MSP. The red aurora (at $630.0 \mathrm{~nm}$ ) has an intensity peak equal to or more than $11 \mathrm{kR}$ during our time of interest. This matches the conditions described by Collis et al. (1991), who reported intensities of 10-30 kR. The precipitation event continues for the rest of our observation time, which stopped at 08:00 UT, but with less intensity after about 07:15 UT. The intensity of the $557.7 \mathrm{~nm}$ and $844.6 \mathrm{~nm}$ lines is more irregular and the events do not last as long as the $630.0 \mathrm{~nm}$ signature. A detailed check of the MSP data is not possible, as the data was lost due to a power failure, and only the "quick-look" plot presented in Fig. 7 is available. Therefore, it is not conclusive whether harder precipitation, represented by the $557.7 \mathrm{~nm}$ emission, is present during our event.

In the analysis of the standard EISCAT data (not shown) around 06:46 UT, when our enhanced echoes are seen, an increase in the electron density also at lower altitudes (below $200 \mathrm{~km}$ ) is noticeable. This is accompanied by an apparent increase in the electron temperature, ion upflow and no apparent increase in the ion temperature. The spectra during this event are clearly non-thermal, and the resulting parameters from the standard analysis should only be treated as apparent.

The ionospheric conditions described above resemble those previously reported in conjunction with enhanced echoes (Sedgemore-Schulthess and St.-Maurice, 2001, and references therein), and although such conditions seem typical, they are also quite unspectacular.

\section{The observations}

In this section we summarise the main features of the observed event. The time scale at which the event evolves, the distribution in range and size of its localised features and in what way the enhanced radar echoes correlates with visual auroral activity are all decided.

\subsection{Time variability}

Before we can decide what integration time we want to employ, we need to know the time scales on which the phenomenon varies. As previous observations have shown significant variability between successive $10 \mathrm{~s}$ (Rietveld et al., 1991) or even $5 \mathrm{~s}$ periods (Rietveld et al., 1996), this time scale has not previously been known, except that it must be shorter than 5 s. Rietveld et al. (1996, Fig. 5b) shows an example of predominantly downshifted enhancement in one $5 \mathrm{~s}$ period, followed by predominantly upshifted enhancement in the next, and hypothesize that even shorter integrations will show even faster changes between enhanced shoulders of opposite Doppler shifts.

In Fig. 8, a section of our data from towards the end of our event is shown, processed using three different integration periods: 50, 100 and $200 \mathrm{~ms}$. The graph shows the maximum power observed in any upshifted or downshifted range gate between 490 and $598 \mathrm{~km}$. From this figure it is obvious that enhancement varies significantly on time scales shorter than a second, but also that simultaneous enhancement in both shoulders occurs even on the shortest time scales, and is not the result of temporal averaging. In the present work, we will use $200 \mathrm{~ms}$ integrations, as this gives sufficient energy for 


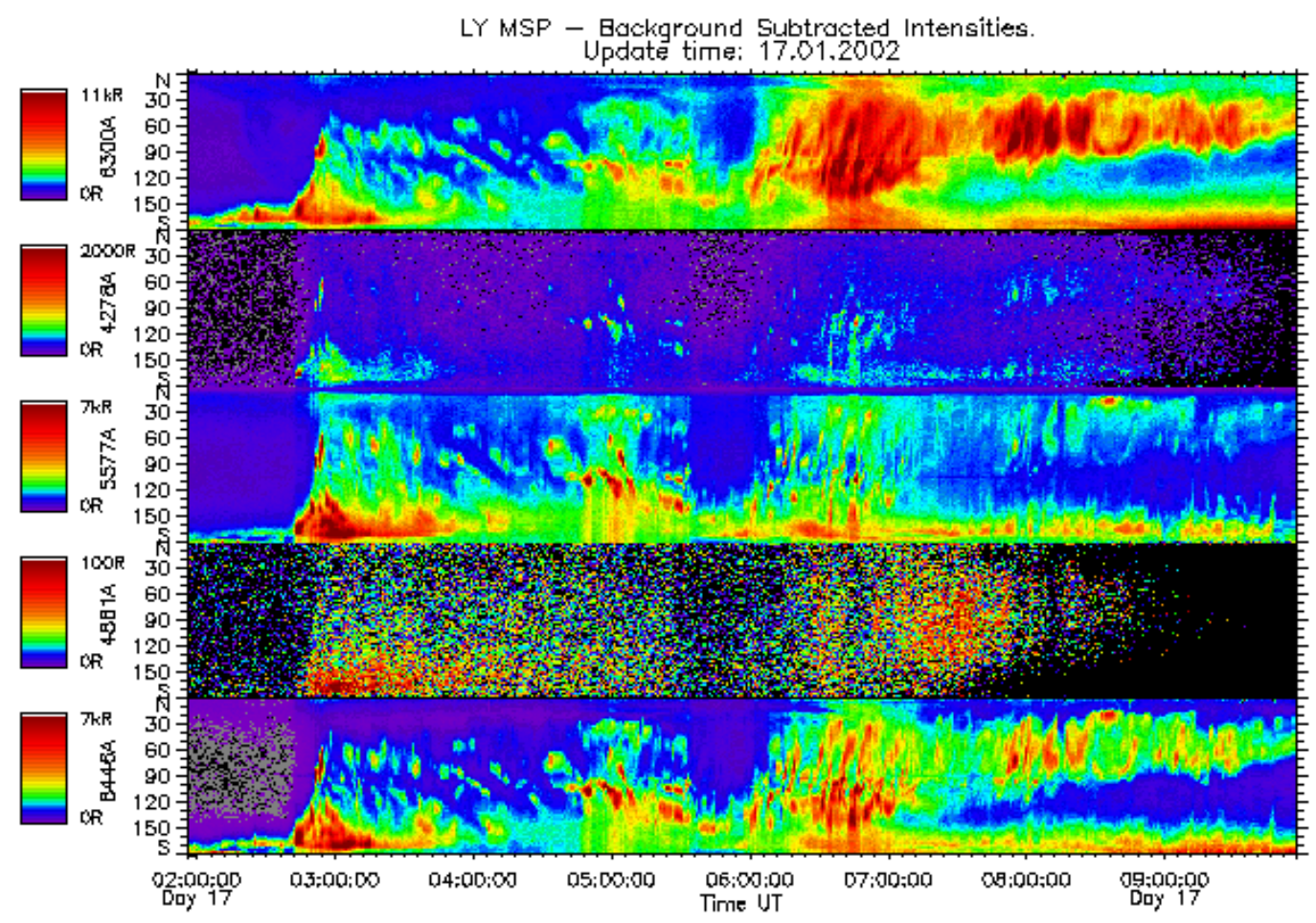

Fig. 7. Meridian scanning photometer data from 02:00-10:00 UT, 17 January 2002. The panels show the $630.0 \mathrm{~nm}, 427.8 \mathrm{~nm}, 557.7 \mathrm{~nm}$, $488.1 \mathrm{~nm}$ and $844.6 \mathrm{~nm}$ auroral emissions from top to bottom, respectively. In the absence of other optical observations, the $630.0 \mathrm{~nm}$ panel gives the impression of a stable red arc which has been associated with enhanced ion-acoustic echoes in earlier studies.

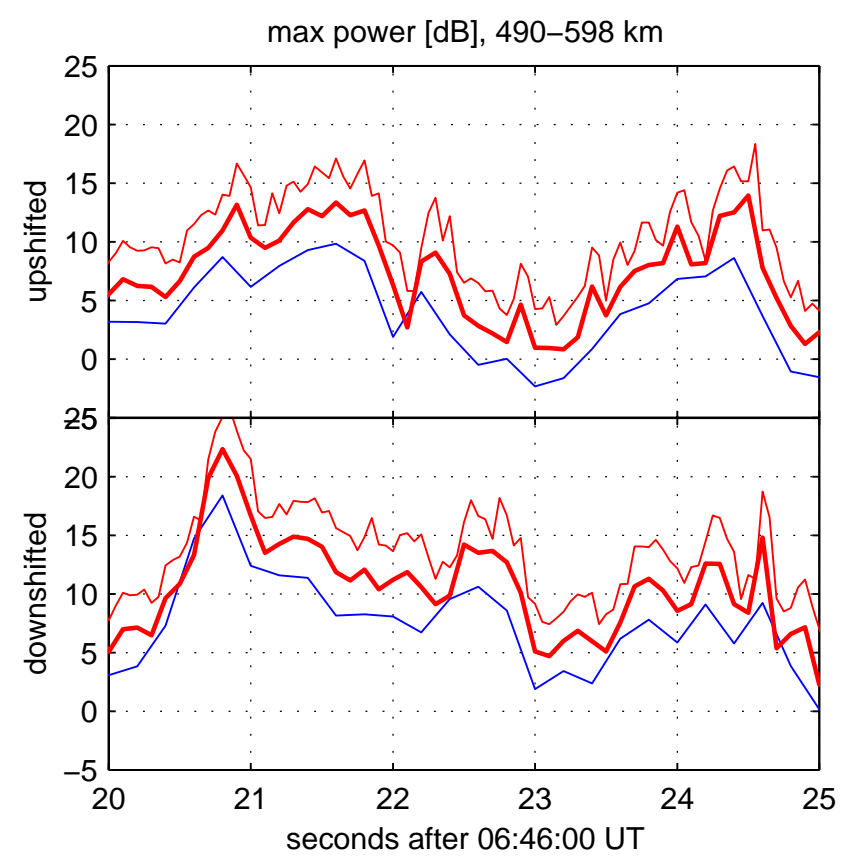

Fig. 8. Comparison of different integrations. The data is processed using three different integration periods: 50, 100 and $200 \mathrm{~ms}$. Then, the maximum up- and down-shifted power per unit range and unit frequency within the range interval $490-598 \mathrm{~km}$ is extracted for each integration period, in order to obtain an idea of how the peak intensity varies with time. The $100 \mathrm{~ms}$ curve has been raised by $3 \mathrm{~dB}$ and the $50 \mathrm{~ms}$ curve by $6 \mathrm{~dB}$, to keep them apart. spectral estimation while capturing the overall dynamics and development of the event. In Fig. 9 we present powerspectra from successive $0.2 \mathrm{~s}$ integrations of data collected from the $42 \mathrm{~m}$ antenna, which shows how the event develops in range and frequency on sub-second timescales.

\subsection{Range distribution}

The distribution over range of all enhanced echoes in our observed event is shown in Fig. 10. This figure should be compared with Fig. 5 in Rietveld et al. (1991). We see a similar pattern of up- and down-shifted enhancement, although the altitude where the number of occurrences is equal in each shoulder is higher - in this case, more than $500 \mathrm{~km}$ compared to the $350-400 \mathrm{~km}$ reported by Rietveld et al. $(1991,1996)$. We must stress that our results are from a single observed event, and that the high number of occurrences here is simply due to a much higher temporal resolution than what has previously been available.

We have not investigated the distribution of vertical extent of the individual enhancements, but the events shown here indicate that enhancement is extended vertically, often over hundreds of kilometres, even when the $57 \mathrm{~km}$ pulse length is taken into consideration.

\subsection{Spatial patterns - filamented structure}

Before the results presented by Grydeland et al. (2003) and in the present work, the best estimate of the horizontal scale 


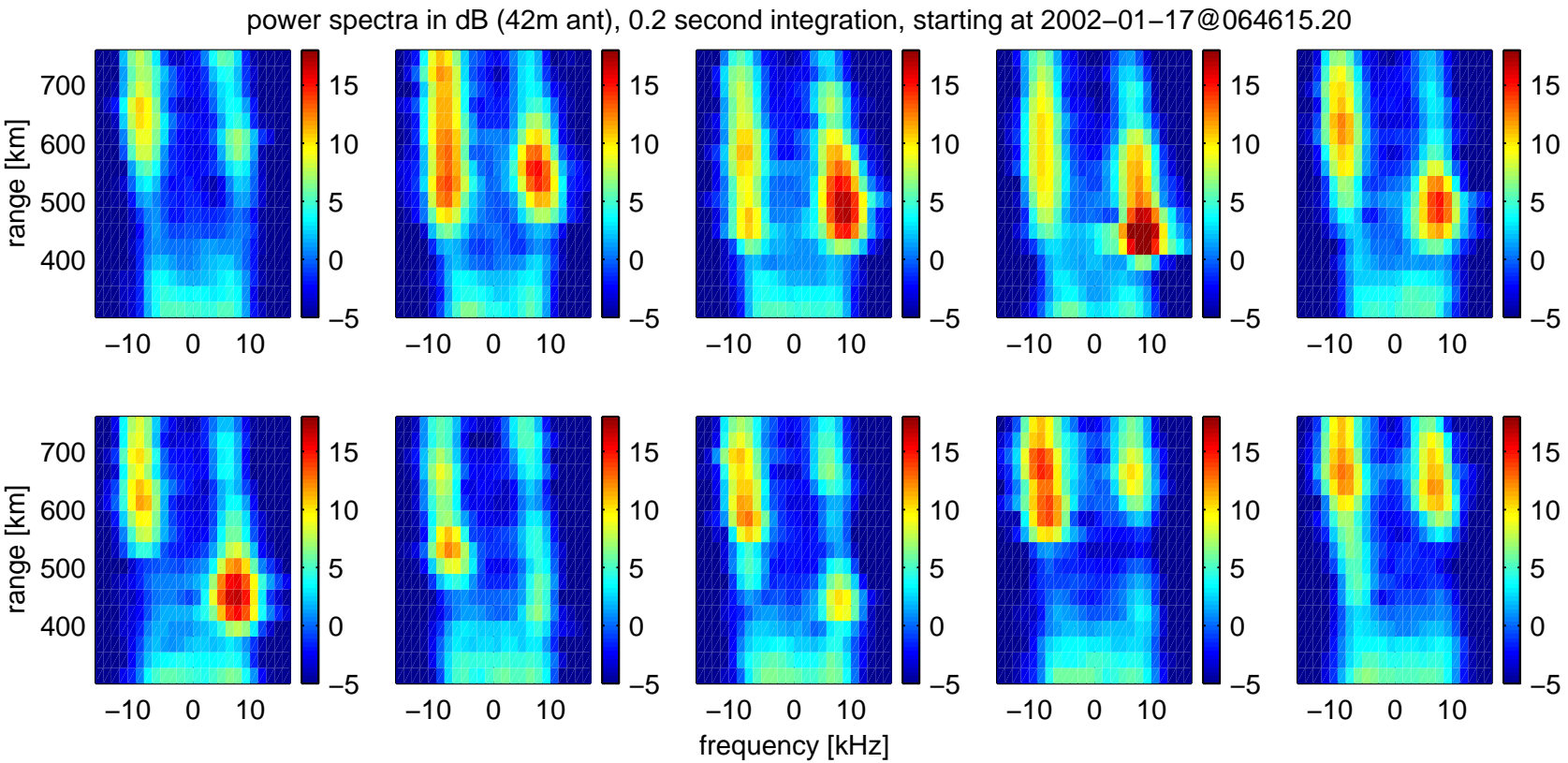

Fig. 9. Example of how an event develops in time. Each panel shows powerspectra from the $42 \mathrm{~m}$ antenna, in $\mathrm{dB}$, on the same scale for successive $0.2 \mathrm{~s}$ integration periods, 2 seconds total. The background noise has not been subtracted, and range ${ }^{2}$ attenuation has not been corrected for. We see for example how one range-frequency region $(+10 \mathrm{kHz}$ at $450 \mathrm{~km})$ shows an increase in scattering by $15 \mathrm{~dB}$ in 0.6 seconds, and then fades back to its original value within the next second or so.

of these phenomena was provided by Rietveld et al. (1996), who used observations at an angle with the magnetic field to conclude that "the extent of the echoing region [...] is very narrow compared to $22 \mathrm{~km}$ ".

As discussed by Grydeland et al. (2003), Eq. (12) lets observed coherence be interpreted in terms of a maximum horizontal structure size. Horizontal scattering structures will not produce a measurable coherence unless they are smaller than the radar beam by a factor of two or more, and scatter strongly enough to dominate relative to the sum of background noise and thermal (incoherent) scatter from the rest of the volume.

For the duration of our event, the range-frequency grid from each $0.2 \mathrm{~s}$ integration period is divided into six regions: up- and down-shifted (ignoring Doppler shifts lower than $5 \mathrm{kHz}$ to reject most satellites), below $\sim 415 \mathrm{~km}$, between 415 and $630 \mathrm{~km}$ and above $630 \mathrm{~km}$. In each sector, the maximum value of coherence was found. This value was then accepted as meaningful, if the following three conditions were fulfilled: 1) at least six grid cells in the $3 \times 3$ grid centered on this point showed a coherence of at least 3/2 standard deviations higher than the mean and at least half as high a coherence as the maximum, 2) at least six cells had phase within $\pi / 4$ of the value in the point with maximum coherence, and 3 ) counting the number of points under 1) and under 2) and summing gave at least 14 points. This is a very conservative condition, which has not produced a single false positive when applied to data from quiet conditions, but which rejects some cases which might have been accepted upon visual inspection. An example is shown in Fig. 11.

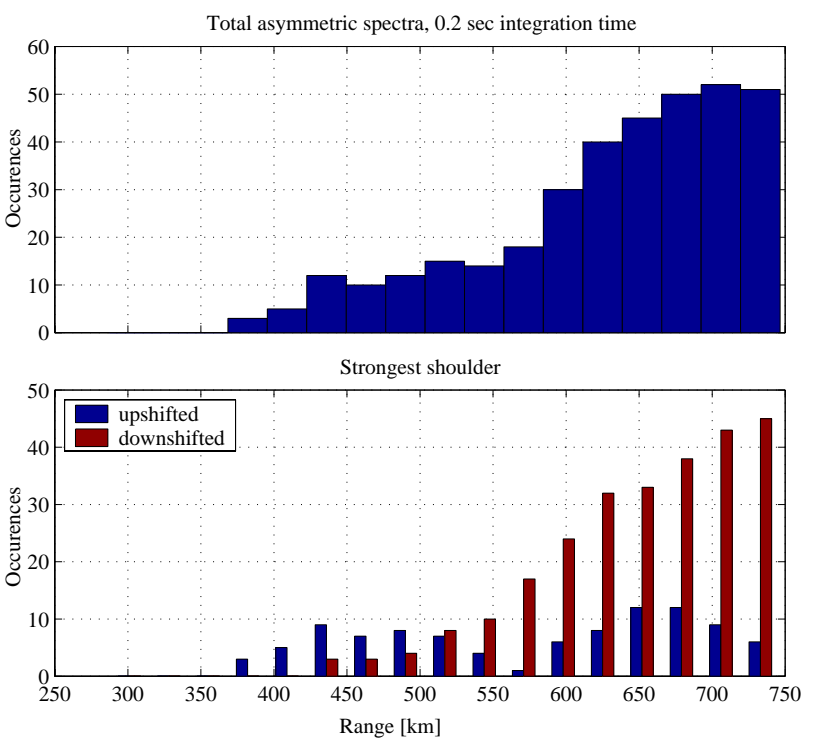

Fig. 10. Histograms showing occurrence of all observed coherent structures vs. range, showing their range and size. The top panel shows all events counted together, while the bottom panel counts up- and down-shifted enhancement separately.

All the accepted coherent structures have been shown in Fig. 12a, plotted versus range and maximum horizontal size. The cut off seen in the figure appears because of the background noise floor, which only allows structures below a certain angular size to be seen, but the corresponding horizontal size is range dependent. Similarly, the trend toward larger 
size structures at longer ranges might be an effect of a lower signal-to-noise ratio, resulting in lower coherence.

As the sizes plotted in Fig. 12a are upper bounds, we should be careful not to try a too detailed interpretation. A few observations can still be made. First, there is no obvious difference between up- and down-shifted enhancements in their distribution in size and range. Second, there is a comparable number of up- and down-shifted enhancements at longer ranges, while Fig. 10 shows larger numbers of downshifted enhancements at longer ranges. It might seem that downshifted enhancement, while more common, is less likely to give rise to observable coherence.

As pointed out by Grydeland et al. (2003), the extremely localised scattering structures in these observations mean that not only is the total scattering increased by a factor of 100 or so, but this increased scattering originates from as little as $0.3 \%$ of the total beam volume, possibly even less. These authors conclude that the scattering cross section within the filament must, therefore, be 4-5 orders of magnitude above thermal levels.

We want to add the observation that observable coherence is in itself an indication that our time resolution is sufficient. If our enhanced scattering were caused by several extremely short-lived structures (simultaneous or not, coherent or not) within an integration, these would have randomly distributed phases, and would not ordinarily result in an observable coherence at longer integrations.

\subsection{Phase as an indication of horizontal position}

The cross-spectrum phase due to a localised scatterer depends on its position along the baseline, as indicated by Eq. (10), and is computed frequency by frequency. As a result, when the coherence indicates a localised scatterer at a given range and frequency, the phase in this range-frequency region can be used to determine the position of the scatterer in one spatial dimension, with some limitations discussed in Sect. 9.1. This information is used to decide whether simultaneous enhancements in up- and down-shifted shoulders occur in the same volume; therefore, from the coherent cases shown in Fig. 12a, those where an upshifted and downshifted coherent enhancement was seen within $100 \mathrm{~km}$ of range of each other in the same $0.2 \mathrm{~s}$ integration were selected. For these cases, the phase in the upshifted enhancement $\left(\phi_{+}\right)$is plotted against the phase in the downshifted enhancement $\left(\phi_{-}\right)$in Fig. 12b. The error bars indicate one standard deviation of phase in the $3 \times 3$ region centered on the point of highest coherence.

The selection criteria used for coherent echoes are quite strict. Furthermore, up- and down-shifted enhancements are considered separately, so occasionally one shoulder or the other is not accepted by the algorithm, although the pattern is quite obvious to a human observer.

Another feature sometimes seen is phase varying linearly with range, consistent with a scatterer located on a field line with its foot-point outside the antenna. If, in such a situation, the search algorithm finds points at slightly different ranges in the two shoulders, they will have a phase difference mainly due to their difference in range. An example of this is shown in Fig. 11, where the performance of the search algorithm is illustrated. The two points in the lowest ranges are both rejected, the other two upshifted coherent points are accepted, while the remaining downshifted points are rejected, in this case because several of the surrounding points are less than $3 / 2$ standard deviations above the mean coherence. Clearly, more sophisticated algorithms are desirable, but even this simple method yields enough evidence to illustrate our point quite clearly.

Even with this restrictive search method, a handful of occurrences were found. Most of these place themselves nicely on the $\phi_{+}=\phi_{-}$line, except for a couple of points where the up- and down-shifted enhancements are clearly unrelated. Two manually identified events (one of which is taken from Fig. 11) have been added; these are drawn in red in this figure. (Two of the other points in the figure have such tiny error bars that they appear more as rectangles than as crosses.)

Our observation that simultaneous up- and down-shifted enhancement is predominantly caused by enhancement on the same or closely neigbouring field lines is contrary to the interpretation by Sedgemore-Schulthess et al. (1999), who, in a similar set-up, use rapid variations in the optical intensity to question the idea that two enhanced shoulders ever coexist on one magnetic field line.

\subsection{Optical observations}

The event in question (17 January 2002, 06:46:1006:46:30 UT) occurred in the middle of an auroral activation, lasting from about 06:00 UT to 07:00 UT. The overall activity was, as mentioned earlier, moderate, but the aurora was dynamic, and appeared sporadically during the activation. A diffuse and very weak arc was present prior to the event, approximately east-west aligned. Around 06:45:55 UT the aurora intensified in the west. The intensification spread quickly, and assumed a rayed appearence, which was characteristic for the event, with thin rays (less than a $\mathrm{km}$ at $105 \mathrm{~km}$ altitude) changing their lower altitude extent almost periodically (approximately $0.5 \mathrm{~s}$ period). Sporadic intensification moves up and down the rays, and also horizontally, across from ray to ray, which gives the auroral display a very dynamic appearance. The observed appearence is typical for so called flaming aurora (Omholt, 1971).

The tall and thin rays mentioned here seem to fit the observations made by Collis et al. (1991) and SedgemoreSchulthess et al. (1999), although close comparison is made difficult due to different instrument resolutions. Figure 13 shows the auroral intensity variations along the interferometry baseline (line a in Fig. 5) during the event. It becomes clear that the detailed picture shown in Fig. 13 (which only summarises the activity captured on the high resolution video system) reveals a lot of activity that can easily be hidden in a lower resolution measurement, like that of the MSP, shown in Fig. 7. 

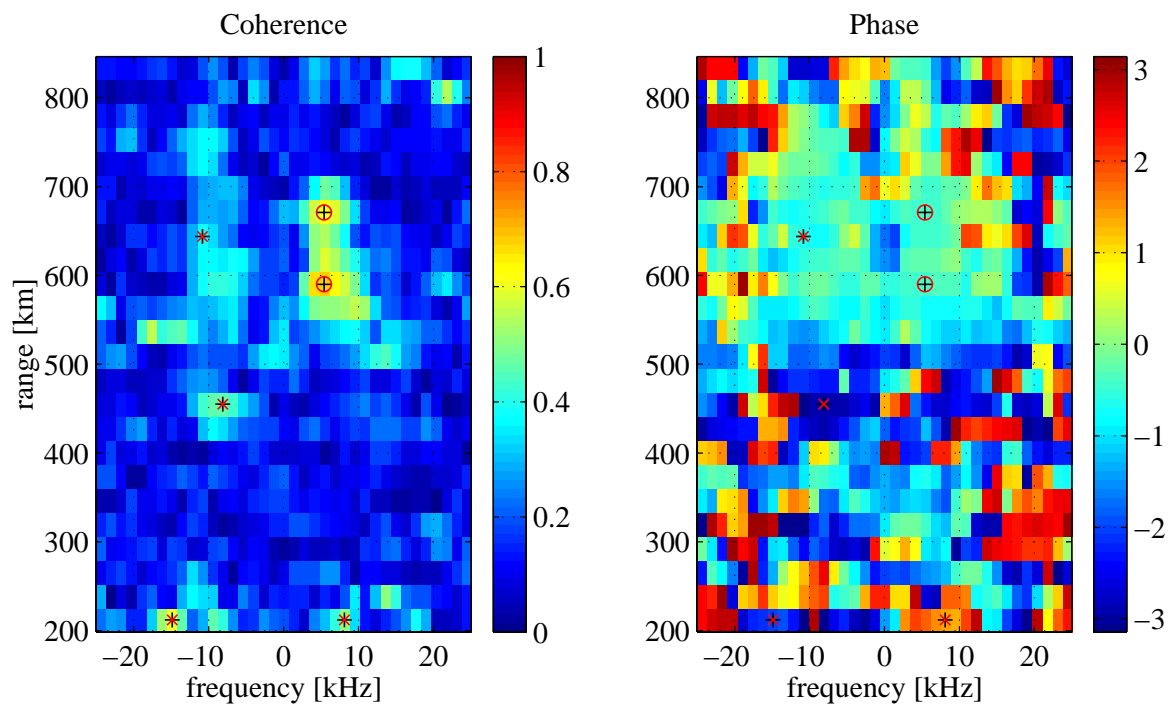

Fig. 11. An example of equal phase in both shoulders not captured by our technique, this is the $0.2 \mathrm{~s}$ integration starting at 06:46:15.60 UT. The two panels show coherence and phase, and the points investigated by the search algorithm are marked with a red circle when accepted, and with a red $\mathrm{x}$, otherwise.
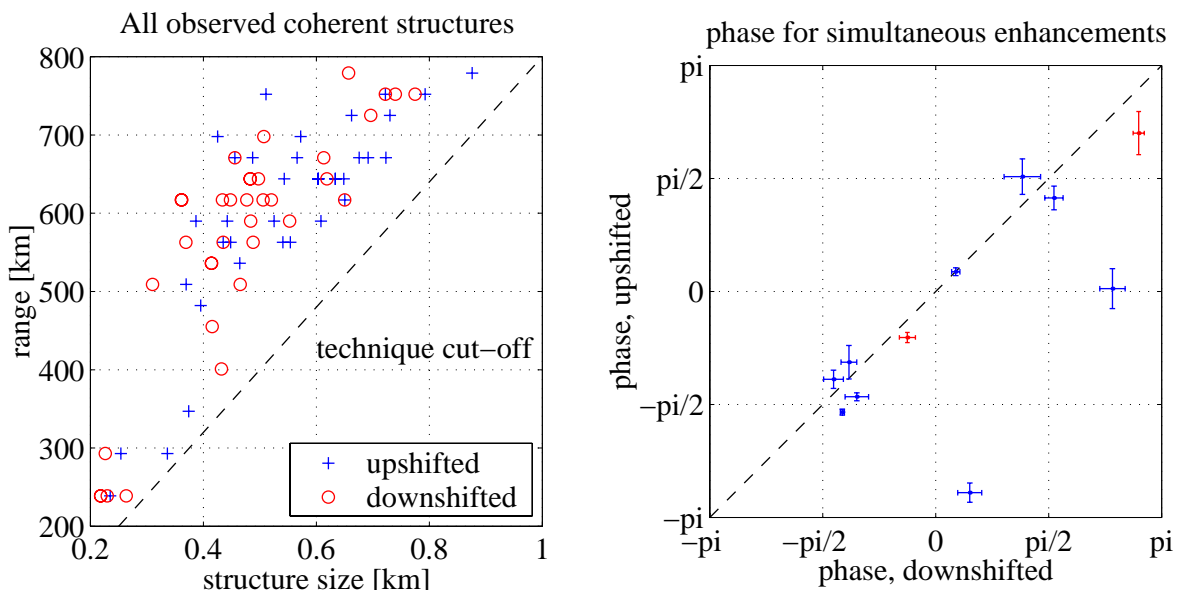

Fig. 12. (a) (left panel) All observed coherent structures, plotted versus range and maximum structure size. (b) (right panel) Phase in the coherent upshifted enhancement versus phase in the coherent downshifted enhancement.

As is evident, most of the auroral activity occurs outside the radar beam (indicated by the horizontal lines in Fig. 13). However, at about 06:46:15 and 06:46:20-24 UT, aurora occurs inside the radar beam. These times coincide roughly with the periods of enhancement seen by the radar. The longest continuous period of activity is shown in Fig. 8, while a similar chart showing the entire event can be found as Fig. 1 of Grydeland et al. (2003).

To explore the relation between the enhanced echoes and auroral activity, simple time series (06:46:10 to 06:46:30 UT) were extracted from the two data sources, which were then cross correlated. In the images from the auroral video camera, two lines are drawn through the projection of the radar beam to $105 \mathrm{~km}$ altitude. These lines are shown in Fig. 5 . Line a corresponds to the interferometer baseline, line $b$ is drawn perpendicular to the baseline, and there is no other general motivation behind the orientation of these lines. In addition to the region centered at the beam, labelled c, two regions along line $b$ were selected, one 25 pixels nearly straight north of the radar beam b1 and one 25 pixels nearly straight south of it b2, each 5 pixels wide. These regions are drawn in red in Fig. 5. From each region the normalised light intensity (per pixel, arbitrary units) were extracted as a function of time during the event. These three time series were then cross correlated with time series extracted from the radar data. To obtain simple time series from the radar data, a method similar to that described in Sect. 8.3 was used. Range-frequency space was divided into four regions, above and below $529 \mathrm{~km}$ in range, and positive/negative Doppler shifts. Then the power at the peak of the shoulder in each 


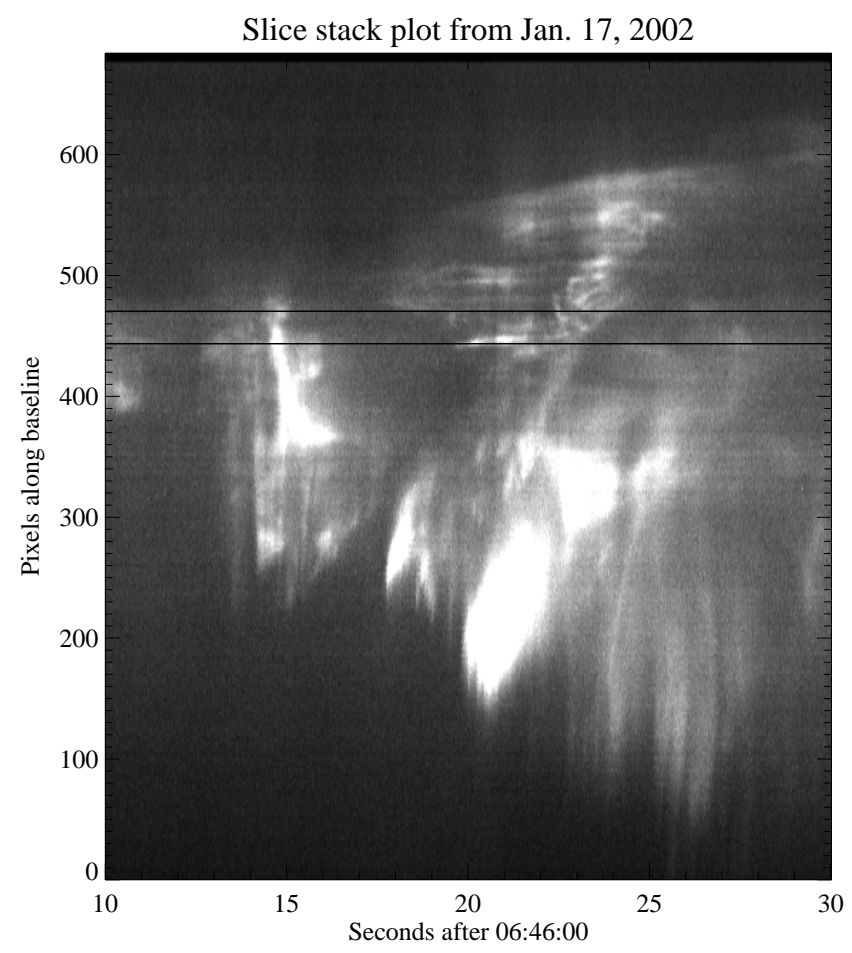

Fig. 13. The auroral activity during the event shown as a slicestack plot (also referred to as a "keogram"). The intensity along the baseline (line a in Fig. 5) is plotted vertically as a function of time, the two horizontal lines shows the position of the radar beam at $105 \mathrm{~km}$.

region was extracted, yielding in all four time series from the radar, in arbitrary units. As the camera data has 30 measured points per second, while the radar only has 5 , the light intensity time series were resampled to attain the same length as the radar time series.

A comparable analysis was made for line a in Fig. 5. The results were similar, except that the analysis along line $b$ was less cluttered and showed slightly higher cross-correlations, which is why only those results are discussed here.

The cross-correlation, $\rho_{R I}(\tau)$, between the radar power time series $(R)$ and the imager data $(I)$, is defined such that for positive lags $(\tau>0), R$ is ahead of $I$. This means that an event which first occurs in the radar time series, and later in the imager data, will result in a positive correlation at a positive lag.

Figure 14 shows the resulting cross-correlation coefficient. The first thing to be noticed is that the correlation coefficient approaches zero at lags greater than $\pm 10 \mathrm{~s}$. Second, it is evident that the overall correlation is rather low, and the highest cross-correlation coefficient $(\sim 0.8)$ appears in Fig. 14 in the lower panel at $\tau \sim-0.8 \mathrm{~s}$. The imager time series used in that cross-correlation is taken from region b1 in Fig. 5. In that region, the geometry is quite simple, similar to that in the idealised Fig. 6.

To reach an approximate estimation of the validity of the cross-correlation the white-noise test was applied (Shiavi, 1999), where the null hypothesis are two uncorrelated Gaus-
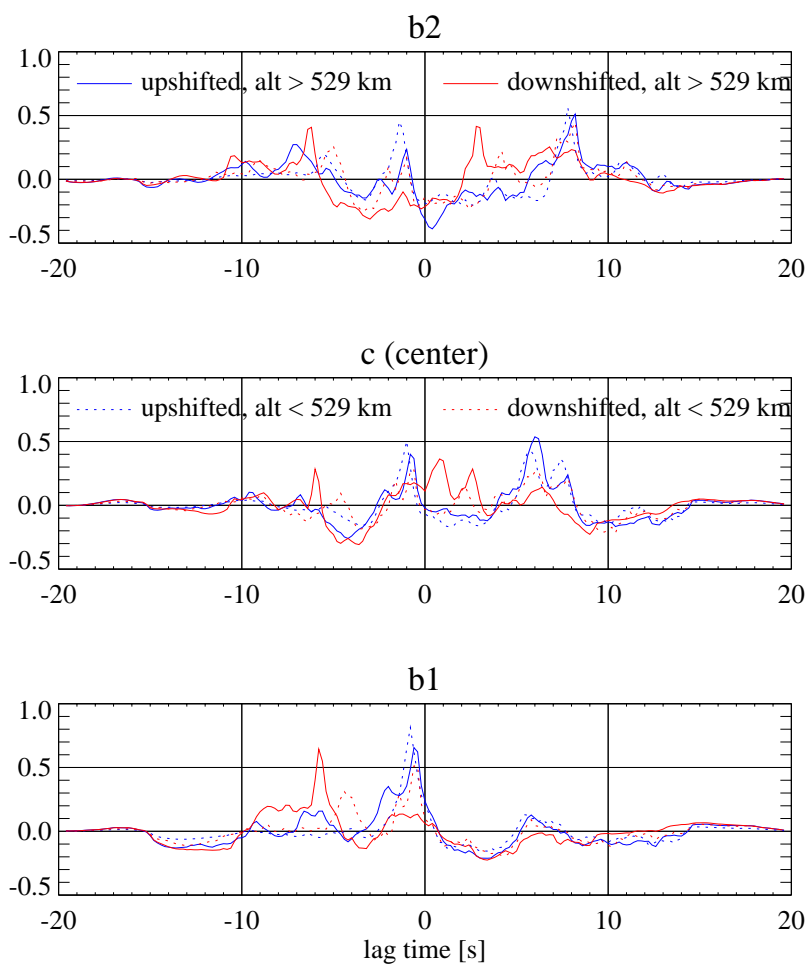

Fig. 14. Cross-correlation between the time series extracted from the radar (for the blue solid line the radar time series is maximum power in the upshifted shoulder above $529 \mathrm{~km}$ altitude) and the camera (the camera time series are normalised light intensity drawn from the one of three regions; b1, c, b2, along line b in Fig. 5). The upper panel shows the cross-correlation between the radar time series and the normalised light intensity from region b2. The middle and lower panels are similar, but the normalised light intensities are taken from the center of the radar beam (region c), and 25 pixels below it (region b1), respectively. Positive lag times correspond to events appearing first in the radar measurements.

sian processes. The $95 \%$ confidence interval for zero correlation then ranges from \pm 0.20 , which means that the statistical significance of the data is acceptable. Thus, the confidence limit for the peak correlation in Fig. 14 is $0.6-1.0$.

A further visual inspection of the data, through a scatter plot of the optical versus radar time series, as shown in Fig. 15, was then done. The normalised light intensity from region $\mathrm{b} 1$, delayed by $0.8 \mathrm{~s}$, is plotted on the $\mathrm{x}$-axis, versus the maximum power (also in arbitrary units) of the upshifted spectra below $529 \mathrm{~km}$ altitude on the y-axis. This plot shows that there is a correlation between the two time series when the normalised light intensity is above a certain threshold (say $\sim 300$ ), while there is a large, uncorrelated population below this threshold.

\section{Discussion}

Observations prior to Grydeland et al. (2003) of naturally enhanced ion-acoustic echoes have used a temporal and spatial resolution which has not fully resolved the dynamics of the 


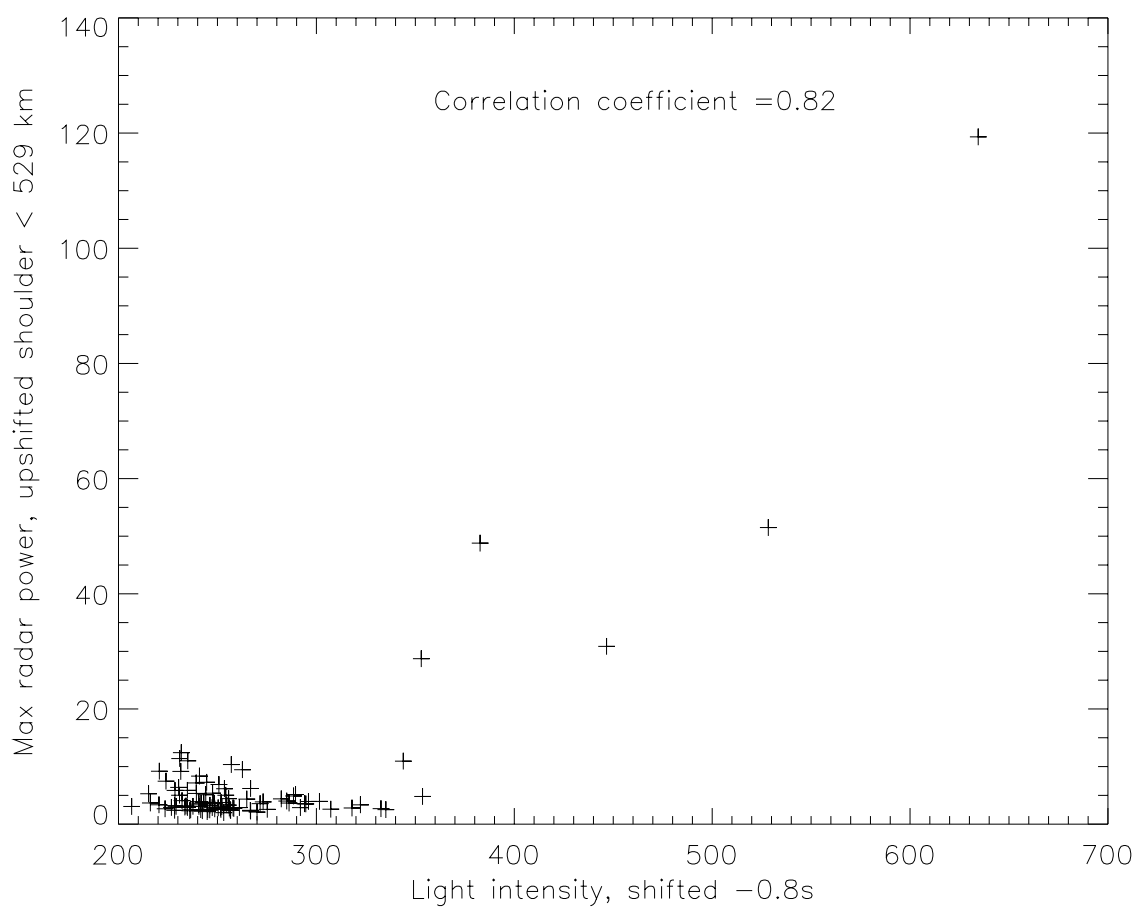

Fig. 15. Scatter plot of normalised light intensity (arbitrary units) versus maximum power (arbitrary units) in the upshifted shoulder of the received radar spectrum (taken below $529 \mathrm{~km}$ ). The light intensity stems from the time series taken from region b1 in Fig. 5 , delayed by $0.8 \mathrm{~s}$.

phenomenon. In this section, we discuss the implications our high resolution measurements have on the understanding of these enhancements, and their relation to auroral activity.

\subsection{The evidence for simultaneous up- and down-shifted enhancements}

As the summary of existing theoretical explanations shows, it is a decisive issue whether or not simultaneously observed up- and down-shifted enhancements actually occur in the same volume and at the same time or not, as some theories will explain enhancement in only one or the other ionacoustic shoulder, while simultaneous enhancement of both is explained in terms of temporal or spatial averaging.

With our technique, the temporal evolution of the event is resolved. We also note that observing coherence at all is inconsistent with an explanation involving enhancements from several unrelated scatterers. Therefore, we conclude without a doubt that when both ion-acoustic shoulders are observed to be enhanced, this occurs simultaneously. The question then becomes whether the simultaneous enhancement occurs within a single volume, or if opposite shoulders are enhanced in nearby volumes within the same beam.

With a single baseline, a localised scatterer detected through interferometry cannot be localised in the direction perpendicular to the baseline, and although the position within any one fringe can be determined through the crossspectrum phase, it is not possible to determine within which fringe it is positioned, due to the phase wrapping around ev- ery $2 \pi$ radians. Consequently, the scatterer cannot be positioned unambiguously along the baseline.

Hence, when both up- and down-shifted enhancements occur within the same integration period, and both have significant coherence and the same cross-spectrum phase, there are at least two ways this can occur without the scatterers occupying the same physical space. The scatterer can be displaced in the direction perpendicular to the baseline - the $\mathrm{y}$-direction - or it can be displaced by a distance in the $\mathrm{x}$ direction, which corresponds to a phase shift of exactly $2 \pi$, or a combination of both.

Both of these explanations cause the same phase to appear by chance. As Fig. 11 illustrates, there are instances of unrelated simultaneous enhancement of both shoulders, and with unrelated phase, but the trend is clearly for the phases to be equal, or very nearly so. Such obvious ordering will not arise by chance.

9.2 On the relation between enhanced echoes and auroral activity

In Sect. 8.5 a correlation between auroral activity and enhanced ion-acoustic echoes appears to have been found, on a time scale which matches the natural time scales of both phenomena.

The scatter plot in Fig. 15 shows two populations, one below a threshold in normalised light intensity of about 300 (arbitrary units) which is uncorrelated, and another population above the threshold which exhibits a clear correlation. This indicates that the auroral intensity (which corresponds 
roughly to plasma production rate) needs to exceed a threshold before the most intensively enhanced ion-acoustic echoes are produced.

In Fig. 14 there are several features worthy of attention: i) The upshifted lines, at low and high altitudes, correlate very similarly, i.e. the blue solid and hatched lines follow each other closely in all panels. This means that the correlation of the upshifted shoulder to auroral activity is nearly altitude independent. ii) A clear peak in the cross-correlation coefficient, with a timelag of about $-0.8 \mathrm{~s}$, is seen in all panels for the upshifted shoulder. This means that auroral activity in any of the three regions along line b in Fig. 5 is followed approximately a second later by an enhanced upshifted echo. This is also true for the same analysis done along line a, which is not shown. Because the auroral activity is so dynamic, changing rapidly on sub-second time scales, it is most likely prompt emissions that we see. Thus, the time lag is not likely contaminated by the lifetime of slow emissions. iii) There is a clear peak in the correlation with the downshifted shoulder for high altitudes at $\tau \sim-6 \mathrm{~s}$. Notice that point ii above supports the notion from the scatter plot (Fig. 15) that it is the auroral intensity that leads, or controls, the enhanced echoes.

The above pattern in the correlation coefficient, the clear peak at $\tau \sim-0.8 \mathrm{~s}$, where $\rho_{R I}(\tau) \sim 0.8$, together with earlier findings that enhanced ion-acoustic echoes coincide with tall rays and coronal structures, suggest that there is a correlation between the two activities.

The lower panel of Fig. 14 seems best suited for an interpretation, as it is taken from a region nearly straight north of the radar beam, and thus has a simple geometry - similar to that shown in Fig. 6 - and it is in that panel that the highest correlation is found. The following scenario is possible: First, an auroral activation becomes visible just north of the radar, about a second later a downward propagating ion-acoustic wave is seen in all altitude regions nearly simultaneously. Approximately $5 \mathrm{~s}$ later an upward propagating wave is activated at higher altitudes. A line drawn through the camera and region b1 (shown in Fig. 5) will intersect the field line whose foot-point is at the radar site at an altitude of $110-115 \mathrm{~km}$. This means that the auroral activity seen in region b1 in Fig. 5 might come from within the radar beam at an altitude of $110-115 \mathrm{~km}$, instead of north of it at an altitude of $105 \mathrm{~km}$.

It should be stressed again that to determine the close relation between the radar and optical events with less ambiguity, one needs to remove the effects of the unfortunate geometry of the experiment. This can most easily be done by colocating the camera with the radar, which in this case was not possible due to problems with interference from the radar in the optical equipment.

The aurora was very dynamic during the event, even though a quick look at MSP data might give the impression of a stable red arc being present. Other studies (Collis et al., 1991; Sedgemore-Schulthess et al., 1999) have concluded that their enhanced radar echoes arise at the border of a red arc, but in the data presented here, "an arc" can hardly be defined. It is not clear whether their data stem from events which are completely different from the one presented here, or if their imaging system could have missed the detailed activity within the aurora. Furthermore, for the type of auroral activity which has been presented here, trying to pinpoint the relative location of aurora and radar backscattering using a time integration longer than $1 \mathrm{~s}$ becomes nearly meaningless.

\subsection{The implications on theories}

We have clearly shown that the enhanced scattering is in fact due to highly localised structures, elongated along the magnetic field, that are sometimes smaller than one percent of the observed volume. Correspondingly, the scattering cross section inside the filament must be 4-5 orders of magnitude higher than in the thermal case. Such enhancement cannot be explained by a mere increase in thermally excited fluctuations through reduced damping, an instability is required. The suggestion by Collis et al. (1991), Rietveld et al. (1991) and others that large (but stable) currents carried by thermal electrons be the mechanism behind the observed enhancement must be rejected. An explanation of our observations involving this mechanism must have thermally carried current densities larger than the threshold for ion-acoustic instability, currents even larger than those (already problematic) suggested previously.

Except for the stable situations discussed by Collis et al. (1991) and by Wahlund et al. (1992), (for the low and high relative drifts), the proposed theories cannot tell what kind of scattering to expect in an unstable situation. The linear theory used to infer spectra for ion-ion and ion-electron twostream instabilities is of little use in a turbulent situation, and the Langmuir turbulence modelling by Forme (1999) does not contain the thermal fluctuation-dissipation situation, so the strength of the turbulent scattering relative to that of the thermal situation cannot be determined.

Another clear result is that simultaneously observed upand down-shifted shoulders are in fact produced simultaneously, and predominantly on the same or immediately neighbouring magnetic field lines. While the ion-ion two-stream enhancement and Langmuir turbulence theories can explain such observations, the ion-electron two-stream enhancement theory must also explain why highly localized and extremely intense field-aligned currents carried by the thermal electron population often - perhaps even usually - arise in opposite directions within a few hundreds of metres of each other.

\section{Conclusions}

The observations reported in this paper are from high resolution measurements of enhanced ion-acoustic echoes, using the EISCAT Svalbard Radar as an interferometer, coordinated with an auroral imager.

The technique employed allows for the radar integration time to be chosen to match the strength and lifetime of the scatterer, even down to a handful of pulses. A post- 
integration of $200 \mathrm{~ms}$ is shown to resolve the time evolution of the enhanced echoes.

The interferometric technique provides upper bounds on the horizontal size of the scattering region of enhanced echoes. Refinements on former techniques used for radar interferometry, including the finite aperture of the antennas in the calculation of the spatial cross-correlation of the scattered signal, gave insignificant improvements in the resulting scale size estimates. The tiny horizontal dimensions deduced in many of the scattering structures implies a scattering cross section inside the structures 4-5 orders of magnitude above thermal levels, which cannot be explained in terms of reduced damping rates - instabilities must be present.

By comparing the cross-spectrum phase of scattering from upward and downward propagating waves received simultaneously (within $200 \mathrm{~ms}$ ) and from the same range, we have shown that these are predominantly closely colocated, confirming previous results (Grydeland et al., 2003).

These two results mean that the ion-electron two-stream enhancement mechanism is an unlikely candidate for explaining these observations. It produces only one enhanced shoulder at a time, so a mechanism which predominantly produces oppositely directed and extremely localised flows of thermal electrons in the immediate vicinity (hundreds of metres) of each other must be provided. Despite the "instability" label often used for this explanation, it is usually discussed for stable configurations. For the enhancement levels reported here, current densities sufficiently high to trigger instability are required - even higher than those inferred previously.

The theories of ion-ion two-stream instability (Wahlund et al., 1992) and cascading Langmuir turbulence theory (Forme, 1999) are both theories involving instability, and which explain enhancement of both shoulders simultaneously. These models seem more likely candidates to explain our findings.

Earlier attempts to correlate the dynamics of enhanced radar echoes with auroral activity have in general concluded that such echoes are situated on the edge of a red arc. In the observations presented here, however, the very dynamic auroral activity made it difficult to even identify an auroral arc, and meaningless to try to pinpoint the positions of discrete auroral structures relative to the region of enhanced scattering on time scales longer than $1 \mathrm{~s}$, even if MSP by itself could give the impression of a stable red arc. It was due to the very high time resolution of the radar experiment that the detailed relations between auroral activity and enhanced echoes could be investigated, and a definite correlation has been established.

The results indicate that the up- and down-shifted enhanced shoulders respond differently to auroral activity. Notice that this is not in contradiction with the findings of simultaneous enhancements of the two, as far as all enhancements occur that close in space and time. The temporal correlation between the enhanced echoes and auroral activity suggests that the auroral activity is leading the evolution, with possibilities of misinterpretation due to the parallax. After an au- roral intensification, which needs to exceed a certain threshold in luminosity, the upshifted ion-acoustic shoulder is enhanced, nearly simultaneously at high and low altitudes, with a delay of about $0.8 \mathrm{~s}$. After a further $5 \mathrm{~s}$ the downshifted shoulder becomes enhanced at altitudes above $529 \mathrm{~km}$.

Acknowledgements. The authors wish to thank Einar Mjølhus for extensive and elucidating discussions on the subject of RF- and beam-enhanced Langmuir turbulence. We also want to thank our late friend and colleague Nikita Shumilov, then at UNIS, for providing us with what MSP data was recovered, and Yngve Birkelund for suggesting and explaining windowed spectral estimation.

We acknowledge the support of the Natural Sciences and Engineering Research Council of Canada by means of a Discovery Grant to L. L. Cogger. The EISCAT Scientific Association is supported by Centre National de la Recherche Scientifique of France, MaxPlanck-Gesellschaft of Germany, Particle Physics and Astronomy Research Council of the United Kingdom, Norges Forskningsråd of Norway, Naturvetenskapliga Forskningsrådet of Sweden, Suomen Akatemia of Finland and the National Institute of Polar Research of Japan.

This project has been supported through grants 120150/431(TG) and 147769/431 from the NFR of Norway.

Topical Editor M. Lester thanks J. Semeter and another referee for their help in evaluating this paper.

\section{References}

Buchert, S. C., van Eyken, A. P., Ogawa, T., and Watanabe, S.: Naturally enhanced ion-acoustic lines seen with the EISCAT Svalbard Radar, Adv. Space Res., 23, 1699-1704, 1999.

Cabrit, B., Opgenoorth, H., and Kofman, W.: Comparison between EISCAT UHF and VHF backscattering cross section, J. Geophys. Res., 101, 2369-2376, 1996.

Chau, J. L. and Woodman, R. F.: Interferometric and dual-beam observations of daytime spread- $F$-like irregularities over Jicamarca, Geophys. Res. Lett., 28, 3581-3584, 2001.

Collis, P. N., Häggström, I., Kaila, K., and Rietveld, M. T.: EISCAT radar observations of enhanced incoherent scatter spectra; their relation to red aurora and field-aligned currents, Geophys. Res. Lett., 18, 1031-1034, 1991.

Farley, D. T., Ierkic, H. M., and Fejer, B. G.: Radar interferometry: A new technique for studying plasma turbulence in the ionosphere, J. Geophys. Res., 86, 1467-1472, 1981.

Forme, F. R. E.: A new interpretation on the origin of enhanced ion acoustic fluctuations in the upper ionosphere, Geophys. Res. Lett., 20, 2347-2350, 1993.

Forme, F. R. E.: Parametric decay of beam-driven Langmuir wave and enhanced ion-acoustic fluctuations in the ionosphere: a weak turbulence approach, Ann. Geophys., 17, 1172-1181, 1999.

Forme, F. R. E., Fontaine, D., and Wahlund, J. E.: Two different types of enhanced ion acoustic fluctuations observed in the upper ionosphere, J. Geophys. Res., 100, 14 625-14 636, 1995.

Foster, J. C., del Pozo, C., Groves, K., and St. Maurice, J.-P.: Radar observations of the onset of current driven instabilities in the topside ionosphere, Geophys. Res. Lett., 15, 160-163, 1988.

Groves, K. M., Lee, M. C., and Foster, J. C.: Nonlinear ionospheric propagation effects on UHF radio signals, in Physics of Space Plasmas (1991), SPI Conference Proceedings, vol. 11, Scientific Publishers, Inc., 377-392, 1991. 
Grydeland, T., Mjølhus, E. Bjørnå: Simultanous observations of enhanced ion spectra and strong plasma lines with the EISCAT UHF radar system, presented at the EGS XXIII General Assembly, Nice, France, 1998.

Grydeland, T., Strømme, A., van Eyken, T., and La Hoz, C.: The capabilities of the EISCAT Svalbard Radar for inter-hemispheric coordinated studies, Chinese J. Pol. Sci., 13, 55-66, 2002.

Grydeland, T., La Hoz, C., Hagfors, T., Blixt, E. M., Saito, S., Strømme, A., and Brekke, A.: Interferometric observations of filamentary structures associated with plasma instability in the auroral ionosphere, Geophys. Res. Lett., 30, 1338, doi:10.1029/2002GL016362, 2003.

Hanssen, A., Mjølhus, E., DuBois, D. F., and Rose, H. A.: Numerical test of the weak turbulence approximation to ionospheric Langmuir turbulence, J. Geophys. Res., 97, 1207312 091, 1992.

Holt, J. M., Erickson, P. J., Gorczyca, A. M., and Grydeland, T.: MIDAS-W: a workstation-based incoherent scatter radar data acquisition system, Ann. Geophys., 18, 1231-1241, 2000.

Kudeki, E., Bhattacharyya, S., and Woodman, R. F.: A new approach in incoherent scatter $F$ region $\mathrm{E}$ x B drift measurements at Jicamarca, J. Geophys. Res., 104, 28 145-28 162, 1999.

Maggs, J. E. and Davis, T. N., Measurements of the thicknesses of auroral structures, Planet. Space Sci., 16, 205-209, 1968.

Nygrén, T.: Introduction to incoherent scatter measurements, Invers Publications, Sodankylä, Finland, 1996.

Omholt, A.: The Optical Aurora, Springer-Verlag, Berlin, 1971.

Percival, D. B. and Walden, A. T.: Spectral analysis for physical applications, Cambridge University Press, Cambridge, England, 1993.

Rietveld, M. T., Collis, P. N., and St.-Maurice, J.-P.: Naturally enhanced ion acoustic waves in the auroral ionosphere observed with the EISCAT 933-MHz radar, J. Geophys. Res., 96, 1929119305, 1991.
Rietveld, M. T., Collis, P. N., van Eyken, A. P., and Løvhaug, U. P.: Coherent echoes during EISCAT UHF common programmes, J. Atmos. Terr. Phys., 58, 161-174, 1996.

Robinson, P. A.: Nonlinear wave collapse and strong turbulence, Rev. Mod. Phys., 69, 507-573, 1997.

Sedgemore-Schulthess, F. and St.-Maurice, J.-P.: Naturally enhanced ion-acoustic spectra and their interpretation, Surv. Geophys., 22, 55-92, 2001.

Sedgemore-Schulthess, K. J. F., Lockwood, M., Trondsen, T. S., Lanchester, B. S., Rees, M. H., Lorentzen, D. A., and Moen, J.: Coherent EISCAT Svalbard Radar spectra from the dayside cusp/cleft and their implication for transient field-aligned currents, J. Geophys. Res., 104, 24 613-24 624, 1999.

Shiavi, R.: Introduction to Applied Statistical Signal Analysis, Academic Press, San Diego, 1999.

Sigernes, F., Svenøe, T., and Deehr, C. S.: The auroral station in Adventdalen, Svalbard, Chinese J. Pol. Sci., 13, 67-74, 2002.

Trondsen, T.: High spatial and temporal resolution auroral imaging, Ph. D. thesis, University of Troms $\varnothing$, Troms $\varnothing, 1989$.

Trondsen, T. S. and Cogger, L. L.: Fine-scale optical observations of aurora, Phys. Chem. Earth (C), 26, 179-188, 2001.

Turunen, T. and Silén, J.: Modulation patterns for the EISCAT incoherent scatter radar, J. Atmos. Terr. Phys., 46, 593-599, 1984.

Wahlund, J.-E., Forme, F. R. E., Opgenoorth, H. J., Persson, M. A. L., Mishin, E. V., and Volokitin, A. S.: Scattering of electromagnetic waves from a plasma: enhanced ion acoustic fluctuations due to ion-ion two-stream instabilities, Geophys. Res. Lett., 19, 1919-1922, 1992.

Wannberg, G., Wolf, I., Vanhainen, L.-G., Koskenniemi, K., Röttger, J., Postila, M., Markkanen, J., Jacobsen, R., Stenberg, A., Larsen, R., Eliassen, S., Heck, S., and Huuskonen, A.: The EISCAT Svalbard radar: a case study in modern incoherent scatter radar system design, Radio Sci., 32, 2283-2307, 1997.

Zakharov, V. E.: Collapse of Langmuir waves, Sov. Phys. JETP, 35, 908-914, (Engl. translation), 1972. 